\title{
A SEMINUMERICAL APPROACH FOR HEAT DIFFUSION IN HETEROGENEOUS MEDIA: ONE EXTENSION OF THE ANALYTICAL QUADRUPOLE METHOD
}

\section{Olivier Fudym}

Departamento de Ingeniería Mecánica, Universidad de Santiago de Chile, Santiago, Chile

\section{Bruno Ladevie}

Centre Energétique-Environnement, UMR 2392 CNRS, Ecole des Mines d'Albi, France

\section{Jean-Christophe Batsale}

LEPT, UMR 8508 CNRS, ENSAM, Esplanade des Arts et Metiers,

Talence, France

The analytical thermal quadrupole method is suitable for the modeling of multidimensional transient heat diffusion in homogeneous media, especially when applied to multilayered media. Here, we propose a new approach in order to extend the quadrupole frame to heterogeneous media. A seminumerical general solution is proposed for transient heat transfer in finite or semi-infinite media in both axial and radial coordinate systems, when the variation of thermal properties is one-dimensional. The presentation of the method is explained with a 2-D two-layer slab case. Some application examples are then presented from this basic case. The analytical expressions allow deep insight about the physical phenomenon.

\section{INTRODUCTION}

The basic thermal quadrupole formalism is a very efficient method for onedimensional linear heat conduction modeling and calculation in multilayered systems $[1,2]$. For transient conduction in a homogeneous material, a linear intrinsic transfer matrix relates the input and output temperature and heat flux after a Laplace transformation and integral space transforms (Fourier or Hankel). The main advantage of this relationship is to make easy the representation of multilayered systems by multiplying the corresponding quadrupole matrices.

This work was partially funded by the FONDECYT program (Award 1020349) as well as the DICYT Department of the Universidad de Santiago. The authors gratefully acknowledge this support.

Address correspondence to Olivier Fudym, Departamento de Ingeniería Mecánica, Universidad de Santiago de Chile, Av. Lib. Bdo. O’Higgins 3363, Casilla 10233, Santiago, Chile. E-mail: ofudym@, lauca.usach.cl 


\begin{tabular}{|c|c|c|c|}
\hline \multicolumn{4}{|c|}{ NOMENCLATURE } \\
\hline$a$ & thermal diffusivity & $\mathbf{V}$ & temperature vector in the diagonal \\
\hline$A, B, C, D$ & generalized quadripole matrices & & basis \\
\hline$e$ & thickness & $\mathbf{Z}$ & generalized thermal impedance \\
\hline $\mathbf{G}$ & heat capacity diagonal matrix $\times \Delta \mathbf{z}$ & $\Delta \mathbf{z}$ & space step diagonal matrix \\
\hline $\mathbf{I}$ & identity matrix & $\varepsilon$ & porosity \\
\hline $\mathbf{J}_{\mathbf{V}}$ & gradient vector in the diagonal basis & $\rho c$ & volumetric heat capacity \\
\hline$k$ & thermal conductivity & $\tau$ & Laplace-Fourier temperature \\
\hline $\mathbf{K}$ & thermal conductivity diagonal & $\phi$ & heat flux in the $x$ direction \\
\hline & matrix $\times \Delta \mathbf{z}$ & $\underline{\varphi}$ & heat flux density in the $z$ direction \\
\hline$L$ & length & $\bar{\Phi}$ & Laplace heat flux vector versus $x$ \\
\hline$N$ & nodes number & $\psi$ & Laplace-Fourier heat flux \\
\hline $\mathbf{P}$ & eigenvectors matrix & $\boldsymbol{\Omega}$ & diagonal eigenvalues matrix \\
\hline$q$ & heat flux density & & \\
\hline$s$ & Laplace variable & Subscripts & \\
\hline$\underline{T}$ & temperature & $1 ; 2$ & input; output \\
\hline $\bar{T}$ & Laplace temperature & $V$ & relative to eigenvalue basis \\
\hline
\end{tabular}

In the case of heterogeneous materials, numerous analytical solutions have been developed for anisotropic conductive heat transfer in some particular composite materials such as two-layer bodies [3,4]. The orthogonal expansion technique can be used to solve the homogeneous problem of a one-dimensional composite medium of finite thickness as the Laplace transformation is used for infinite and semi-infinite case [5]. The Green's functions approach is proposed in [6] for solving the corresponding one-dimensional nonhomogeneous problem. For those cases, a classical quadrupole multilayer approach would be efficient for solving the problem, especially when some direct relationship between interface temperature and heat flux density taken as "input" and "output" variables are to be found [7].

The boundary-element approach, based on discretized boundary integral equations [8, 9], is helpful when solving heat conduction problems in multidimensional cases. However, this approach is not suitable for heterogeneous media unless the number of interfaces is low. Nevertheless, the boundary-element method exhibits some similarities to the thermal quadrupole formalism, provided that for both techniques the state variables and flux are calculated first on the boundaries, and not necessarily on the entire domain. This is an important point when a direct relationship between boundary temperature and heat flux is sought, such as in the case of inverse methods implementation. Also, for both methods, not gridding the whole domain is of substantial benefit when dealing with infinite or semi-infinite media.

The present study deals with the problem of finding a generalized intrinsic relationship between temperature and heat flux at the boundaries of a heterogeneous medium with one-dimensional varying properties in the layer direction. Such cases are very important for applications to the development of thermal nondestructive evaluation methods by infrared thermography [10]. The extension of the thermal quadrupole formalism is obtained from one-dimensional discretization in the properties variation direction, within a control-volume formulation [11], coupled with the semianalytical solution of the corresponding vectorial differential equation. 
The principle of this new method is first presented in the case of a two-layer basic case of heterogeneity in either Cartesian or radial coordinates, with homogeneous or nonhomogeneou soundary conditions. Some aspects related to the usual analytical quadrupole method will be underlined. Some practical aspects of the use of the resulting functions of matrices will then be explained in the case of simple application examples such as a two-layered medium, a single squared heterogeneity, and a periodic medium.

\section{BASIC CASE: TWO-DIMENSIONAL CONDUCTION IN ONE-DIRECTION, VARYING-PROPERTIES MEDIUM}

In a medium with one-dimensional varying thermal properties, such as the one depicted in Figure 1, two-dimensional transient heat conduction is governed by the following equation:

$$
\frac{k(z)}{x^{p}} \frac{\partial}{\partial x}\left(x^{p} \frac{\partial T}{\partial x}\right)+\frac{\partial}{\partial z}\left(k(z) \frac{\partial T}{\partial z}\right)=(\rho c)(z) \frac{\partial T}{\partial t}
$$

where

$$
p= \begin{cases}0 & \text { slab } \\ 1 & \text { cylinder }\end{cases}
$$

The system is assumed to be isothermal $(T=0)$ at time zero. We consider linear boundary conditions such as

$$
-\left.k(0) \frac{\partial T}{\partial z}\right|_{z=0}+h_{0} T(x, 0, t)=f_{0}(x, t) \quad z=0, x_{1}<x<x_{2}
$$

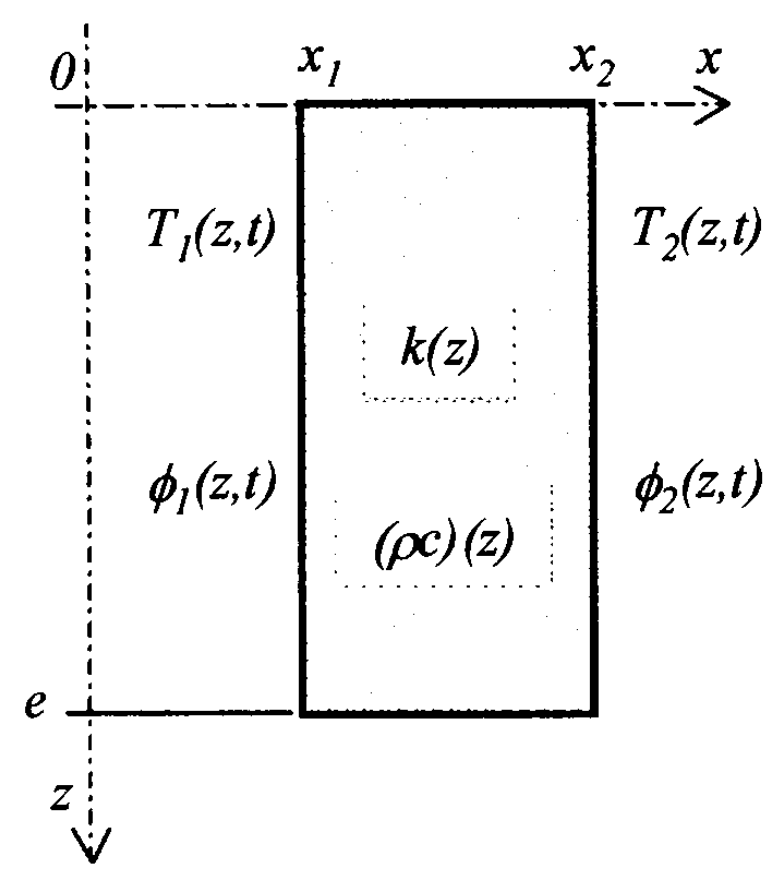

Figure 1. One-dimensional heterogeneous medium. 


$$
\begin{gathered}
\left.k(e) \frac{\partial T}{\partial z}\right|_{z=e}+h_{e} T(x, e, t)=f_{e}(x, t) \quad z=e, x_{1}<x<x_{2} \\
-\left.k(z) \frac{\partial T}{\partial x}\right|_{x=x 1}+h_{x 1} T\left(x_{1}, z, t\right)=f_{x 1}(z, t) \quad x=x_{1}, 0<z<e \\
\left.k(z) \frac{\partial T}{\partial x}\right|_{x=x 2}+h_{x 2} T\left(x_{2}, z, t\right)=f_{x 2}(z, t) \quad x=x_{2}, 0<z<e
\end{gathered}
$$

Space discretization of Eqs. (1) is performed versus the $z$ direction. $N$ new variables are introduced as

$$
T_{i}(x, t)=\frac{1}{\Delta z_{i}} \int_{i^{-}}^{i^{+}} T(x, z, t) d z
$$

where $i^{-}$and $i^{+}$indicate the $i$ th grid interfaces. Equations (1) are then integrated relative to $z$ :

$$
\frac{k_{i} \Delta z_{i}}{x^{p}} \frac{\partial}{\partial x}\left(x^{p} \frac{\partial T_{i}}{\partial x}\right)+\varphi_{i^{-}}-\varphi_{i^{+}}=(\rho c)_{i} \Delta z_{i} \frac{\partial T_{i}}{\partial t}
$$

where $\varphi$ is heat flux density in the $z$ direction. The heat flux $\varphi$ is linearized, and the interface thermal conductances $H_{i^{-}}$and $H_{i^{+}}$are introduced in conservative form:

$$
\begin{aligned}
& H_{i^{-}}=\left(\frac{\Delta z_{i^{-}}}{2 k_{i^{-}} 1}+\frac{\Delta z_{i}}{2 k_{i}}\right)^{-1} \quad \text { and } \quad H_{i^{+}}=\left(\frac{\Delta z_{i}}{2 k_{i}}+\frac{\Delta z_{i+1}}{2 k_{i+1}}\right)^{-1} \\
& \varphi_{i^{-}}=H_{i^{-}}\left(T_{i^{-1}}-T_{i}\right) \quad \text { and } \quad \varphi_{i^{+}}=H_{i^{+}}\left(T_{i}-T_{i^{+} 1}\right)
\end{aligned}
$$

Performing a Laplace transformation for $i=2$ to $N^{-} 1$, such as

$$
\bar{T}_{i}(x, s)=\int_{0}^{\infty} \exp (-s t) T_{i}(x, t) d t
$$

and substituting this expression into Eq. (2b) yields

$$
-H_{i^{-}} \bar{T}_{i^{-1}}+\left[H_{i^{-}}+H_{i^{+}}+(\rho c)_{i} \Delta z_{i} s\right] \bar{T}_{i}-H_{i^{+}} \bar{T}_{i^{+1}}-k_{i} \Delta z_{i} \frac{1}{x^{p}} \frac{d}{d x}\left(x^{p} \frac{d \bar{T}_{i}}{d x}\right)=0
$$

Both boundary conditions at $z=0$ and $z=e$ also have to be expressed in the same way in order to obtain the corresponding equations at node $i=1$ and $i=N$.

For general boundary conditions such as Eqs. $(1 b),(1 c)$, that is, homogeneous or nonhomogeneous ones, the general corresponding equations at nodes 1 and $N$ are given respectively by

$$
\left[c_{1}+H_{1}^{+}+(\rho c)_{1} \Delta z_{1} s\right] \bar{T}_{1}-H_{1}^{+} \bar{T}_{2}-k_{1} \Delta z_{1} \frac{1}{x^{p}} \frac{d}{d x}\left(x^{p} \frac{d \bar{T}_{1}}{d x}\right)=F_{1}(x, s)
$$




$$
-H_{N^{-}} \bar{T}_{N^{-1}}+\left[c_{N}+H_{N^{-}}+(\rho c)_{N} \Delta z_{N} s\right] \bar{T}_{N}-k_{N} \Delta z_{N} \frac{1}{x^{p}} \frac{d}{d x}\left(x^{p} \frac{d \bar{T}_{N}}{d x}\right)=F_{N}(x, s)
$$

where the coefficients $c_{1}, c_{N}, F_{1}$, and $F_{N}$ are given in Table $1 . F_{1}$ and $F_{N}$ are expressed as Laplace transform functions, while $c_{1}$ and $c_{N}$ are real coefficients.

Introducing the vector $\mathbf{T}$ of the $N$ Laplace transformed temperatures at the position $x$, Eqs. (3) can be written in the matrix form

$$
\mathbf{K}^{-1}\left(\mathbf{M}_{/ /}+\mathbf{G} s\right) \overline{\mathbf{T}}-\frac{1}{x^{p}} \frac{d}{d x}\left(x^{p} \frac{d \overline{\mathbf{T}}}{d x}\right)=\mathbf{F}
$$

with

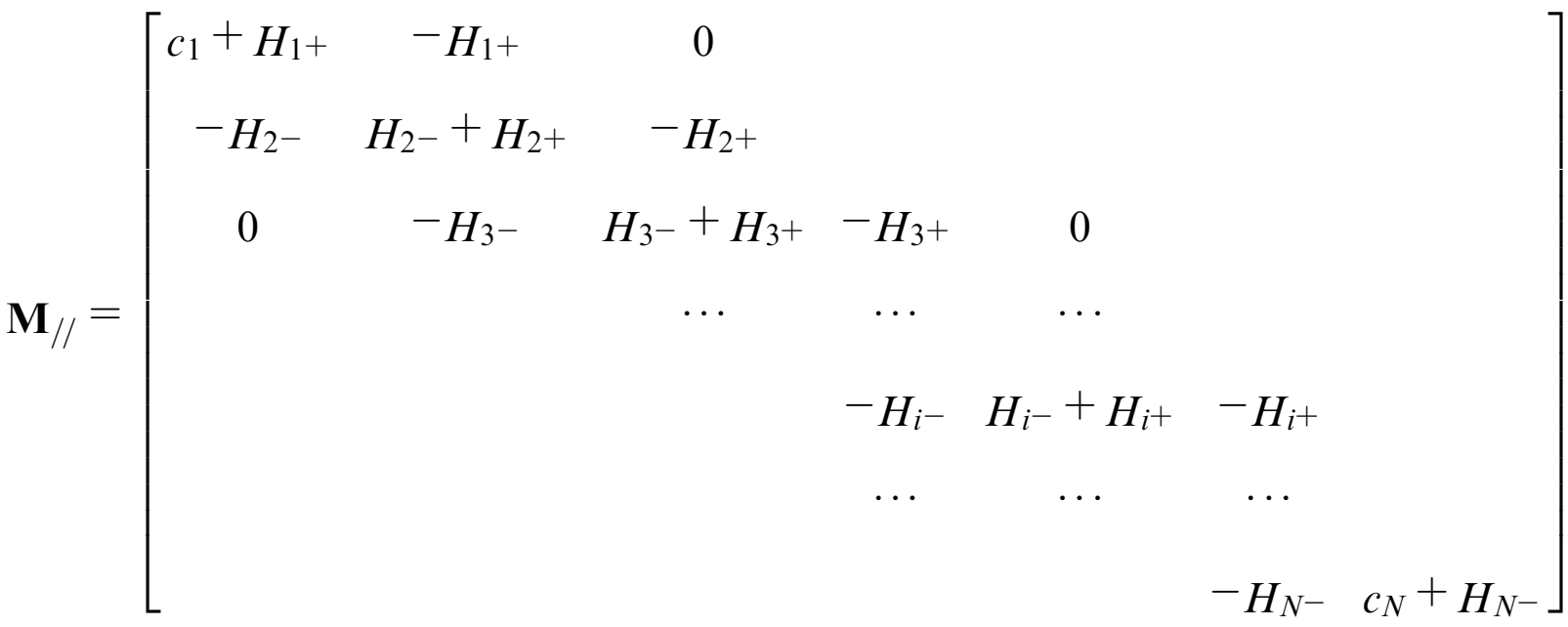

$$
\begin{aligned}
& \mathbf{G}=\operatorname{diag}\left\{\left[(\rho c)_{1} \Delta z_{1}(\rho c)_{2} \Delta z_{2} \cdots(\rho c)_{N} \Delta z_{N}\right]\right\} \quad \mathbf{K}=\operatorname{diag}\left[\left(k_{1} \Delta z_{1} \cdots k_{N} \Delta z_{N}\right)\right] \\
& \overline{\mathbf{T}}=\left[\begin{array}{llll}
\bar{T}_{1} & \bar{T}_{2} & \cdots & \bar{T}_{N}
\end{array}\right]^{t} \text { and } \mathbf{F}=\left[\begin{array}{lllll}
F_{1} & 0 & \cdots & 0 & F_{N}
\end{array}\right]^{t}
\end{aligned}
$$

where the operator diag is used in order to build a diagonal matrix from the corresponding vector.

The square matrix $\mathbf{K}^{-\mathbf{1}}\left(\mathbf{M}_{/ /}+\mathbf{G s}\right)$ on the left side of Eq. (4) is independant of $x$. Equation (4) can be solved directly by the diagonalization of this matrix. The diagonalization of this system yields

\begin{tabular}{|c|c|c|}
\hline B.C. at $z=0$ & $c_{1}$ & $F_{1}$ \\
\hline$T(x, 0, t)=f_{0}(x, t)$ & $\frac{2 k_{1}}{\Delta z_{1}}$ & $\frac{2 k_{k}}{\Delta z_{1}} \overline{f_{0}}(x, s)$ \\
\hline$\varphi(x, 0, t)=g_{0}(x, t)$ & 0 & $\overline{g_{0}}(x, s)$ \\
\hline$\varphi(x, 0, t)=h_{0}[T \infty(x, t)-T(x, 0, t)]$ & $\frac{2 k_{1} h_{0} \Delta}{2 k_{1}+h_{0} \Delta z_{1}}$ & $\frac{2 k_{1} h_{0}}{2 k_{1}+h_{0} \Delta z_{1}} \overline{T \infty}(x, s)$ \\
\hline B.C. at $z=e$ & $c_{N}$ & $F_{N}$ \\
\hline$T(x, e, t)=f_{e}(x, t)$ & $\frac{2 k x}{\Delta z_{x}}$ & $\frac{2 k v}{\Delta_{z}} \overline{f_{e}}(x, s)$ \\
\hline$\varphi(x, e, t)=g_{e}(x, t)$ & 0 & $-\bar{g}_{e}(x, s)$ \\
\hline$\varphi(x, e, t)=h_{e}\left[T(x, e, t)-T_{\infty}(x, t)\right]$ & $\frac{2 k_{x} h_{e}}{2 k_{N}+h_{e} \Delta_{Z_{N}}}$ & $\frac{2 k_{N} h_{c}}{2 k_{N}+h_{c} \Delta z_{N}} \overline{T_{\infty}}(x, s)$ \\
\hline
\end{tabular}

$$
\mathbf{K}^{-1}\left(\mathbf{M}_{/ /}+\mathbf{G} s\right)=\mathbf{P} \Omega \mathbf{P}^{-1}
$$

Table 1. Boundary condition coefficients for $z=0$ and $z=e$ 
where $\boldsymbol{\Omega}$ is a diagonal matrix. Introducing the temperature vector in the new basis,

$$
\mathbf{V}=\mathbf{P}^{-1} \overline{\mathbf{T}}
$$

Equation (4) is then written as

$$
\Omega \mathbf{V}-\frac{1}{x^{p}} \frac{d}{d x}\left(x^{p} \frac{d \mathbf{V}}{d x}\right)=\mathbf{P}^{-\mathbf{1}} \mathbf{F}
$$

It is very important to point out that in Eq. (5c), only the unknown vector $\mathbf{V}$ and boundary condition vector $\mathbf{F}$ can be $x$-dependent.

The operator defined in Eq. ( $5 a)$ can be generalized to a three-dimensional situation. This involves a spatial integral transformation in the third direction ( $y$ or $\theta$ ) if the medium is homogeneous following the third direction and generalizes matrix G. If the medium is nonhomogeneous following the third direction, the gridding is then 2-D (following $x$ and $y$ or $\theta$ ) and only matrix $\mathbf{M}_{/ /}$must be changed.

The last expression $(5 c)$ is a simple second-order ordinary differential diagonal system. Such a system can be solved with functions of matrices. This will be explained in the case of homogeneous or nonhomogeneous boundary conditions. One advantage is then to obtain symbolic expressions in the case of infinite media or asymptotic expansions for instance in the case of infinitely thin layers.

\subsection{Homogeneous Boundary Conditions}

For homogeneous boundary conditions, $\mathbf{F}$ is zero. Each line of Eq. $(5 c)$ can be solved directly in a scalar way, due to the fact that $\boldsymbol{\Omega}$ is a diagonal matrix, and the corresponding equation is homogeneous. The general results can be arranged in a matrix form, applying the quadrupole formalism results. Care must be taken not to commutate the matricial products.

If $\mathbf{F}$ is zero, each line of Eq. $(5 c)$ is

$$
\frac{1}{x^{p}} \frac{d}{d x}\left(x^{p} \frac{d v_{k}}{d x}\right)-d_{k} v_{k}=0
$$

The general solution of Eq. $(6 a)$ has the form

$$
v_{k}=G_{1} H_{1}^{p}(x, s)+G_{2} H_{2}^{p}(x, s)
$$

where the basic functions $H_{1}^{p}$ and $H_{2}^{p}$ depend on the geometry:

- $p=0$, slab:

$$
H_{1}^{0}=\cosh \left(\sqrt{d_{k}} x\right) \quad H_{2}^{0}=\sinh \left(\sqrt{d_{k}} x\right)
$$

or

$$
H_{1}^{\prime 0}=\exp \left(+\sqrt{d_{k}} x\right) \quad H_{2}^{\prime 0}=\exp \left(-\sqrt{d_{k}} x\right)
$$

- $\quad p=1$, cylinder:

$$
H_{1}^{1}=I_{0}\left(\sqrt{d_{k}} x\right) \quad H_{2}^{1}=K_{0}\left(\sqrt{d_{k}} x\right)
$$


where $I_{0}$ and $K_{0}$ are the modified Bessel functions of order zero of the first and second kinds, respectively. The coefficient $d_{k}$ is a function of the Laplace variable $s$, but is independant of $x$.

The scalar thermal quadrupole formalism is derived from the solution of Eq. ( $6 a$ ), by eliminating the integration constants $G_{1}$ and $G_{2}$ in Eq. (6b) in order to find a linear relationship between temperature and heat flux in the Laplace domain [1].

Introducing the flux expressed in the eigenvalues basis as

$$
j_{k}=-S(p) \frac{d v_{k}}{d x} \quad \text { with } S(p)=(2 \pi x)^{p}
$$

the scalar thermal quadrupole at $x_{1}$ and $x_{2}$ location is written in the following form:

$$
\left[\begin{array}{c}
v_{k} \\
j_{k}
\end{array}\right]_{x_{1}}=\left[\begin{array}{ll}
A_{k} & B_{k} \\
C_{k} & D_{k}
\end{array}\right]\left[\begin{array}{l}
v_{k} \\
j_{k}
\end{array}\right]_{x_{2}}
$$

The scalar terms of the quadrupole depend on the geometry [12]. The same quadrupole formalism can now be implemented in a vectorial form. Using vector $\mathbf{V}$ of $\left(v_{k}\right)$ and introducing the flux $\mathbf{J}_{\mathbf{V}}$ as

$$
\mathbf{J}_{\mathbf{V}}=-S(p) \frac{d \mathbf{V}}{d x}
$$

and the equivalent vectorial form of the previous quadrupole expression is

$$
\left[\begin{array}{c}
\mathbf{V} \\
\mathbf{J}_{\mathbf{V}}
\end{array}\right]_{x_{1}}=\left[\begin{array}{ll}
\mathbf{A} \mathbf{V} & \mathbf{B}_{\mathbf{V}} \\
\mathbf{C}_{\mathbf{V}} & \mathbf{D}_{\mathbf{V}}
\end{array}\right]\left[\begin{array}{c}
\mathbf{V} \\
\mathbf{J}_{\mathbf{V}}
\end{array}\right]_{x_{2}}
$$

where $A_{\mathbf{v}}, \mathbf{B}_{\mathbf{v}}, \mathbf{C}_{\mathbf{v}}$, and $\mathbf{D}_{\mathbf{v}}$ are diagonal matrices built with the scalar terms corresponding to Eq. $(6 d)$, as shown in Table 2. Index $V$ refers to the fact that the temperature and heat flux vectors are written in the eigenvalues basis of matrix $\mathbf{K}^{-1}\left(\mathbf{M}_{/ /}+\mathbf{G} s\right)$. In Table 2, some functions of diagonal matrices are written: a function applied to a diagonal matrix is defined as an operator where the function applies to each diagonal element of the matrix.

The boundary conditions following the $x$ direction are given as a relationship between temperature and heat flux such as Eqs. (1d)-(1e), but are unknown in the $V$ basis. It is thus convenient to express $\mathbf{J}_{\mathbf{V}}$ as a function of heat flux vector $\Phi$ :

$$
\overline{\mathbf{\Phi}}=-S(p) \mathbf{K} \frac{d \overline{\mathbf{T}}}{d x}
$$

Table 2. Quadrupole term in the eigenspace for slab and cylinder: $L=x_{2}-x_{1} ; \mathbf{X}_{1}=\sqrt{\mathbf{\Omega}} x_{1} ; \mathbf{X}_{2}=\sqrt{\mathbf{\Omega}} x_{2}$

\begin{tabular}{lll}
\hline Geometry & \multicolumn{1}{c}{$\operatorname{Slab}(p=0)$} & \multicolumn{1}{c}{ Cylinder $(p=1)$} \\
\hline $\mathbf{A}_{\mathbf{v}}$ & $\cosh (\sqrt{\mathbf{\Omega}} L)$ & $\mathbf{X}_{2}\left[\mathbf{I}_{0}\left(\mathbf{X}_{1}\right) \mathbf{K}_{1}\left(\mathbf{X}_{2}\right)+\mathbf{I}_{1}\left(\mathbf{X}_{2}\right) \mathbf{K}_{0}\left(\mathbf{X}_{1}\right)\right]$ \\
$\mathbf{B}_{\mathbf{v}}$ & $\sinh \left(\sqrt{\mathbf{\Omega}} L(\sqrt{\mathbf{\Omega}})^{-1}\right.$ & $\frac{1}{2 \pi}\left[\mathbf{I}_{0}\left(\mathbf{X}_{2}\right) \mathbf{K}_{0}\left(\mathbf{X}_{1}\right)-\mathbf{I}_{0}\left(\mathbf{X}_{1}\right) \mathbf{K}_{0}\left(\mathbf{X}_{2}\right)\right]$ \\
$\mathbf{C}_{\mathbf{v}}$ & $(\sqrt{\mathbf{\Omega}}) \sinh (\sqrt{\mathbf{\Omega}} L)$ & $2 \pi \mathbf{X}_{1} \mathbf{X}_{2}\left[\mathbf{I}_{1}\left(\mathbf{X}_{2}\right) \mathbf{K}_{1}\left(\mathbf{X}_{1}\right)-\mathbf{I}_{1}\left(\mathbf{X}_{1}\right) \mathbf{K}_{1}\left(\mathbf{X}_{2}\right)\right]$ \\
$\mathbf{D}_{\mathbf{v}}$ & $\cosh (\sqrt{\mathbf{\Omega}} L)$ & $\mathbf{X}_{\mathbf{1}}\left[\mathbf{I}_{\mathbf{0}}\left(\mathbf{X}_{\mathbf{2}}\right) \mathbf{K}_{\mathbf{1}}\left(\mathbf{X}_{\mathbf{1}}\right)+\mathbf{I}_{\mathbf{1}}\left(\mathbf{X}_{\mathbf{1}}\right) \mathbf{K}_{\mathbf{0}}\left(\mathbf{X}_{\mathbf{2}}\right)\right]$ \\
\hline
\end{tabular}


The components of the diagonal matrix $\mathbf{K}$ are the thermal conductivity multiplied by the space step of each layer. The components of the heat flux vector $\Phi$ are the heat flux through each layer. Substituting Eqs. (5b) and (7a) into Eq. (8a) yields

$$
\overline{\mathbf{\Phi}}=\mathbf{K} \mathbf{P} \mathbf{J}_{\mathbf{V}}
$$

Using Eqs. (5b) and (8b), it is now possible to express the $V$-form quadrupole given by Eq. $(7 b)$ as a generalized thermal quadrupole in terms of temperature and heat flux vectors, such as

$$
\left[\begin{array}{c}
\mathbf{P}^{-1} \overline{\mathbf{T}} \\
(\mathbf{K P})^{-1} \overline{\mathbf{\Phi}}
\end{array}\right]_{x_{1}}=\left[\begin{array}{ll}
\mathbf{A v}_{\mathbf{V}} & \mathbf{B}_{\mathbf{V}} \\
\mathbf{C}_{\mathbf{V}} & \mathbf{D}_{\mathbf{V}}
\end{array}\right]\left[\begin{array}{c}
\mathbf{P}^{-1} \overline{\mathbf{T}} \\
(\mathbf{K P})^{-1} \overline{\mathbf{\Phi}}
\end{array}\right]_{x_{2}}
$$

and the generalized thermal quadrupole is then

$$
\begin{aligned}
& \mathbf{A}=\mathbf{P} \mathbf{A v}_{\mathbf{V}} \mathbf{P}^{-1} \\
& {\left[\begin{array}{l}
\overline{\mathbf{T}}_{1} \\
\overline{\mathbf{\Phi}}_{1}
\end{array}\right]=\left[\begin{array}{ll}
\mathbf{A} & \mathbf{B} \\
\mathbf{C} & \mathbf{D}
\end{array}\right]\left[\begin{array}{l}
\overline{\mathbf{T}}_{2} \\
\overline{\boldsymbol{\Phi}}_{2}
\end{array}\right]} \\
& \mathbf{B}=\mathbf{P B}_{\mathbf{V}}(\mathbf{K P})^{-1} \\
& \text { with } \\
& \mathbf{C}=\mathbf{K P C}_{\mathbf{V}} \mathbf{P}^{-1} \\
& \mathbf{D}=\mathbf{K P D}_{\mathbf{V}}(\mathbf{K P})^{-1}
\end{aligned}
$$

As an extension of the classical quadrupole formalism, Eq. (9) is a generalized intrinsic relationship between temperature and flux (versus $x$ ) vectors on both boundaries of the corresponding heterogeneous medium. This formalism is useful for multicomponent heterogeneous systems, if only an "input-output" relationship is desired: in that case, the quadrupoles obtained for each element are to be multiplied.

Equation (9) is also valid for the steady-state case, when replacing the matrix $\mathbf{K}^{-1}\left(\mathbf{M}_{/ /}+\mathbf{G} s\right)$ in Eq. (4) by the product $\mathbf{K}^{-\mathbf{1}} \mathbf{M}_{/ / \text {. It is necessary, however, to point }}$ out an important difference from the scalar case: the "determinant" $\mathbf{A D}-\mathbf{B C}$ is not equal to the identity matrix (although $\mathbf{A v}_{\mathbf{V}} \mathbf{D}_{\mathbf{V}}-\mathbf{B}_{\mathbf{V}} \mathbf{C}_{\mathbf{V}}$ is), and also $\mathbf{A} \neq \mathbf{D}$, even if $\mathbf{A}_{\mathbf{v}}=\mathbf{D}_{\mathbf{v}}$. This result implies that the matricial quadrupole cannot be represented through an equivalent generalized electrical impedance approach, except in the eigenvectors space, where the potential is given by vector $\mathbf{V}$, and the current by the gradient vector $\mathbf{J}_{\mathbf{V}}$.

\subsection{Nonhomogeneous Boundary Conditions}

For nonhomogeneous boundary conditions, at $z=0$ and $z=e$, each line of Eq. $(5 c)$ is written as

$$
\frac{1}{x^{p}} \frac{d}{d x}\left(x^{p} \frac{d v_{k}}{d x}\right)+g_{k}=d_{k} v_{k}
$$

where the apparent source term $g_{k}$ represents the $k$ th element of vector $\mathbf{P}^{-\mathbf{1}} \mathbf{F}$.

The solution of Eq. $(10 a)$ has the form

$$
v_{k}=G_{1} H_{1}^{p}(x, s)+G_{2} H_{2}^{p}(x, s)+y(x, s)
$$


where $y(x, s)$ is a particular solution of Eq. (10a). This solution can be obtained from the following expansion [1]:

$$
y_{k}=d_{k}^{-1} g_{k}+d_{k}^{-2} \frac{d^{2} g_{k}}{d x^{2}}+d_{k}^{-3} \frac{d^{4} g_{k}}{d x^{4}}+d_{k}^{-4} \frac{d^{6} g_{k}}{d x^{6}}+\cdots
$$

Equation $(10 c)$ can be expressed in more compact form as

$$
\mathbf{Y}=\Omega^{-1} \mathbf{P}^{-1} \mathbf{F}+\Omega^{-2} \mathbf{P}^{-1} \frac{d^{2} \mathbf{F}}{d x^{2}}+\Omega^{-3} \mathbf{P}^{-1} \frac{d^{4} \mathbf{F}}{d x^{4}}+\cdots
$$

The general input-output relationship in the quadrupole form corresponding to Eqs. $(5 c)$ and $(10 d)$ is then implemented from its scalar equivalent [12], and Eq. (9) for the nonhomogeneous boundary conditions case turns out to be

$$
\left[\begin{array}{l}
\overline{\mathbf{T}}_{\mathbf{1}} \\
\bar{\Phi}_{1}
\end{array}\right]=\left[\begin{array}{ll}
\mathbf{A} & \mathbf{B} \\
\mathbf{C} & \mathbf{D}
\end{array}\right]\left[\begin{array}{l}
\overline{\mathbf{T}}_{\mathbf{2}} \\
\bar{\Phi}_{2}
\end{array}\right]-\left[\begin{array}{l}
\mathbf{X} \\
\mathbf{W}
\end{array}\right]
$$

with

$$
\begin{aligned}
\mathbf{X} & =-\mathbf{P}\left[\mathbf{Y}_{\mathbf{1}}-\mathbf{A V}_{\mathbf{V}} \mathbf{Y}_{\mathbf{2}}+S(p) \mathbf{B}_{\mathbf{V}} \mathbf{Y}_{\mathbf{2}}^{\prime}\right] \\
\mathbf{W} & =(\mathbf{K P})^{-\mathbf{1}}\left[S(p) \mathbf{Y}_{\mathbf{1}}^{\prime}+\mathbf{C}_{\mathbf{V}} \mathbf{Y}_{\mathbf{2}}-S(p) \mathbf{D}_{\mathbf{V}} \mathbf{Y}_{\mathbf{2}}^{\prime}\right]
\end{aligned}
$$

This development is similar to the corresponding homogeneous scalar case [13, Chap. 3, pp. 87-89].

\subsection{Asymptotic Expansions for a Thin Medium}

Functions of matrices consist of a symbolic representation of complex phenomena. One advantage is being able to implement asymptotic expansions in order to obtain simplified expressions. One example can be considered in the case of an infinitely thin medium. In fact, Eq. (4) can be solved for some particular cases by introducing such functions of matrices directly. For instance, in the slab case $(p=0)$, with homogeneous boundary conditions $(\mathbf{F}=\mathbf{0})$, Eqs. (4) and (8a) can be rearranged to

$$
\frac{d}{d x}\left[\begin{array}{c}
\overline{\mathbf{T}} \\
\bar{\Phi}
\end{array}\right]=\left[\begin{array}{cc}
0 & -\mathbf{K}^{-\mathbf{1}} \\
-\left(\mathbf{M}_{/ /}+\mathbf{G} s\right) & 0
\end{array}\right] \cdot\left[\begin{array}{c}
\overline{\mathbf{T}} \\
\bar{\Phi}
\end{array}\right]_{x}
$$

Calculation of the previous system can be implemented by the use of functions of matrices. The formal solution of Eq. (12a) yields

$$
\left[\begin{array}{l}
\overline{\mathbf{T}}(x) \\
\bar{\Phi}(x)
\end{array}\right]=\exp \left(\left[\begin{array}{cc}
0 & -\mathbf{K}^{-1} x \\
-\left(\mathbf{M}_{/ /}+\mathbf{G} s\right)_{x} & 0
\end{array}\right]\right) \cdot\left[\begin{array}{l}
\overline{\mathbf{T}}(0) \\
\bar{\Phi}(0)
\end{array}\right]
$$

and this equation can be written in a quadrupole way between 0 and $L$ as 


$$
\left.\left.\left[\begin{array}{l}
\overline{\mathbf{T}}(0) \\
\bar{\Phi}(0)
\end{array}\right]=\exp \quad \begin{array}{cc}
0 & \mathbf{K}^{-\mathbf{1}} L \\
\left(\mathbf{M}_{/ /}+\mathbf{G} s\right)_{L} & 0
\end{array}\right]\right) \cdot\left[\begin{array}{l}
\overline{\mathbf{T}}(L) \\
\bar{\Phi}(L)
\end{array}\right]
$$

The above example of using a function of matrices such as Eq. (12c) could also apply in a more general frame for Eqs. (9), and some equivalent matrix functions could be defined for the quadrupole matricial terms $\mathbf{A}, \mathbf{B}, \mathbf{C}$, and $\mathbf{D}$. In the case of various longitudinal multilayer slabs, the corresponding matrices are simply multiplied. Direct methods to compute the exponential of a matrix are generally less timeconsuming than classical numerical methods based on gridding versus $x$. However, no full efficient method exists to compute such functions easily. The two main methods [14] consist of the matrix decomposition method (diagonalization of the matrix to be applied to the exponential function) and the asymptotic expansion method (Taylor expansion of the exponential function). The main problem arising with both methods is the ill conditioning of the system, especially with increasing values of $L$.

The Taylor expansion of the exponential function yields, if the length $L$ is low, a first-order approximation with respect to $L$, and a quite simplified model is

$$
\left[\begin{array}{l}
\overline{\mathbf{T}}(0) \\
\bar{\Phi}(0)
\end{array}\right]=\left[\begin{array}{cc}
\mathbf{I} & \mathbf{K}^{-\mathbf{1}} L \\
\left(\mathbf{M}_{/ /}+\mathbf{G} s\right) L & \mathbf{I}
\end{array}\right]\left[\begin{array}{l}
\overline{\mathbf{T}}(L) \\
\bar{\Phi}(L)
\end{array}\right]
$$

The previous expression is quite suitable for the thin heterogeneity case. When a thin insulating layer has to be considered (delamination in a composite multilayered sample), and the inertial and conductive effects in the $z$ direction are negligible, it yields

$$
\left[\begin{array}{l}
\overline{\mathbf{T}}(0) \\
\bar{\Phi}(0)
\end{array}\right]=\left[\begin{array}{cc}
\mathbf{I} & \mathbf{K}^{-1} L \\
\mathbf{0} & \mathbf{I}
\end{array}\right]\left[\begin{array}{c}
\overline{\mathbf{T}}(L) \\
\bar{\Phi}(L)
\end{array}\right]
$$

Thus the local heat flux is conservative.

In the opposite case, if a thin metallic inclusion has to be considered, the temperature continuity is preserved and only a flux redistribution will be added:

$$
\left[\begin{array}{l}
\overline{\mathbf{T}}(0) \\
\bar{\Phi}(0)
\end{array}\right]=\left[\begin{array}{cc}
\mathbf{I} & \mathbf{0} \\
\left(\mathbf{M}_{/ /}+\mathbf{G} s\right) L & \mathbf{I}
\end{array}\right]\left[\begin{array}{l}
\overline{\mathbf{T}}(L) \\
\bar{\Phi}(L)
\end{array}\right]
$$

The asymptotic expansion allows us to avoid computing the eigenvalues and can be used as a direct model. Unfortunately, when $L$ is increased, such an asymptotic expansion is not suitable. On the other hand, the analysis of the problem in the eigenspace can be considered in order to study a semi-infinite medium.

\subsection{Semi-Infinite Medium}

When one of the boundaries of the medium is located toward infinity in the $x$ direction ( $x_{2}$ in Figure 1), the corresponding coefficient $G_{1}$ in the scalar Eq. (6b) has to be zero, in order to get a finite solution. Then, the vector of the constants $G_{2}$ can 
be determined through a particular value, and Eq. (6b) can be written in the matrix form

$$
\mathbf{V}(x)=\mathbf{H}^{p}(\sqrt{\mathbf{\Omega}} \cdot x) \cdot \frac{\mathbf{V}\left(x_{1}\right)}{\mathbf{H}^{p}\left(\sqrt{\Omega} \cdot x_{1}\right)}
$$

where $\mathbf{H}^{p}$ is the exponential function $\exp ()$ if $p=0$ (slab) or the modified Bessel function of order zero of the second kind $K_{0}$ if $p=1$ (cylinder), and $x_{1}$ is any particular point in the semi-infinite medium. According to Eqs. (5b) and (14a), Eq. (8a) can now be expressed, for any $x$, as

$$
\begin{aligned}
& \bar{\Phi}(x)=-S(p) \mathbf{K} \frac{d \mathbf{T}(x)}{d x}=-S(p) \mathbf{K} \mathbf{P} \frac{d \mathbf{V}(x)}{d x} \\
& \bar{\Phi}(x)=-S(p) \mathbf{K} \mathbf{P}^{\sqrt{\Omega}} \cdot x \frac{d \mathbf{H}^{p}(\sqrt{\Omega} x)}{d x} \cdot \frac{\mathbf{V}\left(x_{1}\right)}{\mathbf{H}^{p}\left(\sqrt{\Omega} \cdot x_{1}\right)} \\
& \bar{\Phi}(x)=-S(p) \mathbf{K} \mathbf{P}^{\sqrt{\Omega}} \cdot x \frac{d \mathbf{H}^{p}(\sqrt{\Omega} x) / d x}{\mathbf{H}^{p}\left(\sqrt{\Omega} \cdot x_{1}\right)} \mathbf{P}^{-1} \mathbf{T}\left(x_{1}\right)
\end{aligned}
$$

Equations $(14 a)-(14 b)$ can also be arranged in order to find a direct relationship between the Laplace temperature vector $\mathbf{T}_{1}$ and Laplace heat flux vector $\bar{\Phi}_{1}$ at the position $x_{1}$ :

$$
\overline{\mathbf{T}}_{\mathbf{1}}=-\frac{1}{S(p)} \mathbf{P} \frac{\mathbf{H}^{p}\left(\sqrt{\Omega} \cdot x_{1}\right)}{\sqrt{\Omega} \cdot x_{1}\left(\frac{d \mathbf{d}^{p}}{d x}\right)\left(\sqrt{\Omega} \cdot x_{1}\right)} \mathbf{P}^{-1} \mathbf{K}^{-1} \bar{\Phi}_{\mathbf{1}}=\mathbf{M} \infty \bar{\Phi}_{\mathbf{1}}
$$

The operator $\mathbf{M} \infty$ describing the semi-infinite medium, and defined by Eq. (14c), yields a direct generalized relationship between the input temperature and heat flux vectors of the semi-infinite medium. Depending on the geometry, $\mathbf{M}_{\infty}$ can be expressed as a function of matrices such as

- $p=0$, slab:

$$
\mathbf{M}_{\mathbf{x}}=\mathbf{P} \frac{1}{\sqrt{\Omega}} \mathbf{P}^{-1} \mathbf{K}^{-1}
$$

- $\quad p=1$, cylinder of internal radius $r$ :

$$
\mathbf{M}_{\mathbf{r}}=\frac{1}{2 \pi r} \mathbf{P} \frac{K_{0}(\sqrt{\Omega} r)}{\sqrt{\Omega} K_{1}(\sqrt{\Omega} r)} \mathbf{P}^{-1} \mathbf{K}^{-1}
$$

Such expressions are very efficient compared to classical numerical methods because they avoid gridding in the semi-infinite direction. 


\section{TWO-LAYER SLAB CASE: ANALYTICAL SOLUTION AND VALIDATION}

\subsection{Two-Dimensional Conduction in a Two-Layer Slab}

We consider steady-state conduction in a longitudinal two-layer slab as illustrated in Figure $2 a$. In this case, the analytical methods are difficult to implement, since the use of an integral transform relative to the $z$ coordinate requires numerical calculation of the eigenvalues related to the two-layer heterogeneity.

Assuming, for instance, that the boundary condition at $x=L$ is $\mathbf{T}_{L}=\left[\begin{array}{llll}0 & \cdots & 0 & 0\end{array}\right]^{t}$, both Eqs. (9) and Table 2 yield the temperature field as

$$
\mathbf{T}_{\mathbf{x}}=\mathbf{P}\left\{\sinh [\sqrt{\Omega}(L-x)][\sqrt{\Omega} \cosh (\sqrt{\Omega} L)]^{-1}\right\}(\mathbf{K P})^{-1} \phi
$$

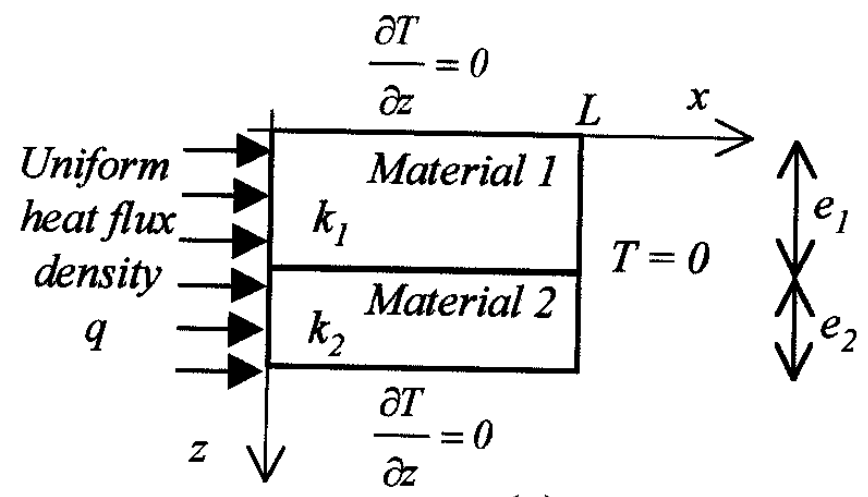

(a)

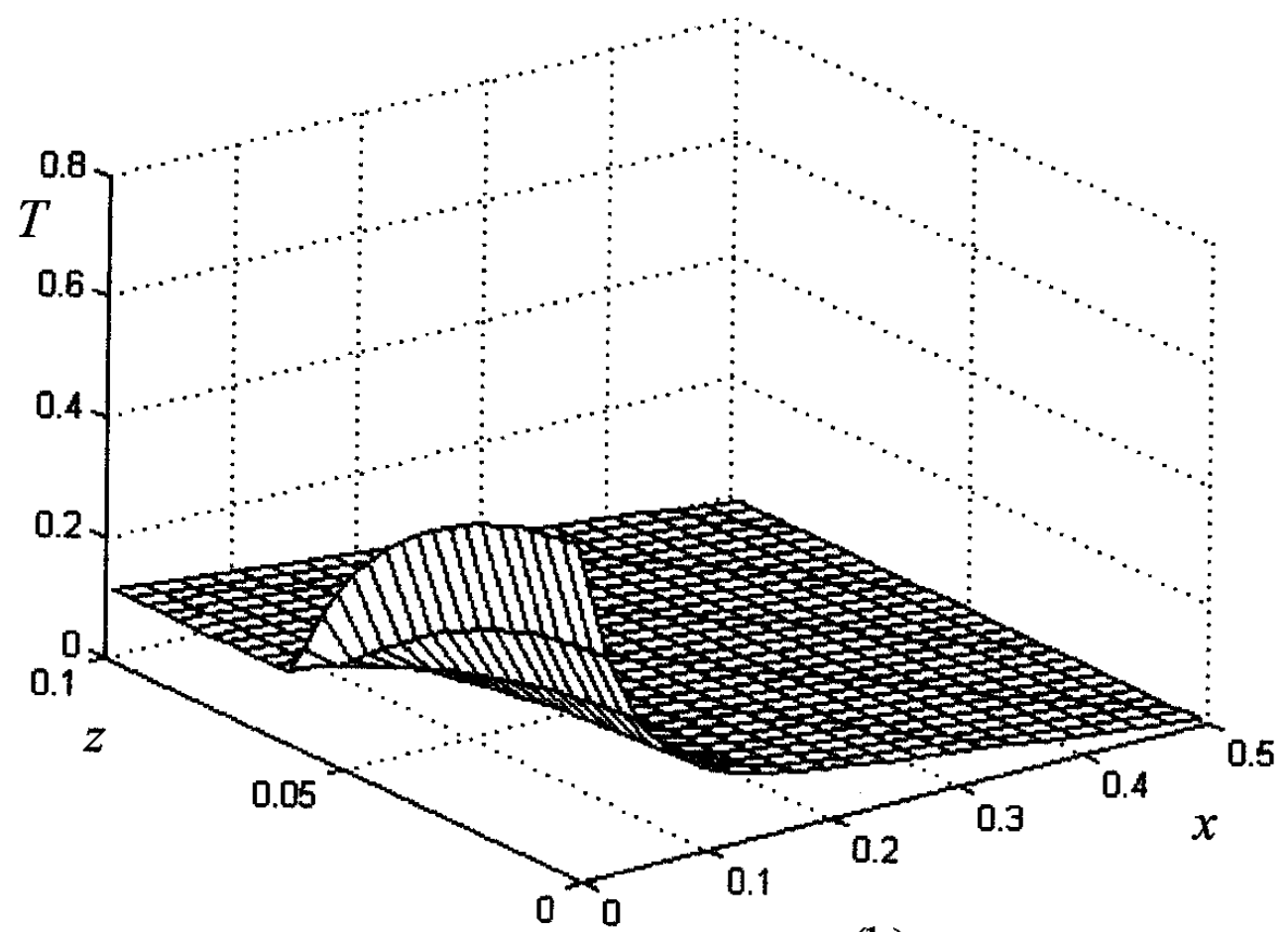

(b)

Figure 2. (a) Two-layer slab; (b) steady-state temperature field $k_{1}=0.1 \mathrm{~W} / \mathrm{m} \mathrm{K}, k_{2}=10 \mathrm{~W} / \mathrm{m} \mathrm{K}$, $L=0.5 \mathrm{~m}, e_{1}=e_{2}=0.05 \mathrm{~m}, N=40$. 
where $\mathbf{T}_{\mathbf{x}}$ is the temperature vector versus the $z$ direction at the $x$ location, and $\phi=q .\left[\begin{array}{lll}\Delta z_{1} & \cdots & \Delta z_{N}\end{array}\right]^{t}$ is the wall heat flux vector.

Such an expression can present some difficulties because the sinh and cosh functions can be undefined by the computer when $L$ tends to infinite or large values. This point is one of the disadvantages related to the use of functions of matrices, such as cosh, sinh, or exp, which may introduce excessively large differences between the lowest and highest eigenvalues of the matrix under consideration. One way to avoid such a problem is to regularize the eigenvalues, and this will be shown in the next example. Here, the input wall temperature vector at $x=0$ can be expressed as

$$
\mathbf{T}_{\mathbf{0}}=\mathbf{P}\left[\frac{\tanh (\sqrt{\Omega} L)}{\sqrt{\Omega}}\right](\mathbf{K P})^{-1} \phi
$$

Equation ( $15 b)$ is stable for any length $L$, because the hyperbolic tangent function is. Both Eqs. (15a) and (15b) also would be suitable for transient transfer, assuming they would be written in the Laplace space. The temperature field computed from Eq. $(15 a)$ is shown in Figure $2 b$.

\subsection{Analytical Solution and Validation for the Two-Layer Slab Steady-State Case}

Solving analytically the previous two-layer slab case in transient state is also possible through an integral transform approach. Applying a cosine Fourier transform on $x$ and Laplace on time to temperature in medium 1 yields the nonhomogeneous equation

$$
\frac{d^{2} \tau}{d z^{2}}-\gamma^{2} \tau+\frac{q}{k_{1} S}=0
$$

where $\tau\left(\beta_{n}, z, s\right)=\int_{0}^{\infty} \int_{0}^{L} T(x, z, t) \exp \left(-{ }_{s t}\right) \cos \left(\beta_{n} x\right) d x d t ; \gamma$ is the generalized frequency $\gamma=\sqrt{\beta_{n}^{2}+s / a_{1}}$ and $\beta_{n}=(2 n+1) \pi / 2 L$ are the discrete eigenvalues corresponding to the boundary-value problem relative to $x$. In the Fourier-Laplace space, a direct linear relationship can be deduced between temperature and heat flux in the $z$ direction, at $z=0$ and $z=e_{1}$ :

$$
\left[\begin{array}{c}
\tau\left(\beta_{n}, z=0, s\right) \\
\psi\left(\beta_{n}, z=0, s\right)=0
\end{array}\right]=\left[\begin{array}{ll}
A_{1} & B_{1} \\
C_{1} & D_{1}
\end{array}\right]\left[\begin{array}{c}
\tau\left(\beta_{n}, z=e_{1}, s\right) \\
\psi\left(\beta_{n}, z=e_{1}, s\right)
\end{array}\right]-\left[\begin{array}{c}
X_{1} \\
Y_{1}
\end{array}\right]
$$

where $X_{1}$ and $Y_{1}$ are obtained from a particular solution of Eq. (16) and $A_{1}, B_{1}, C_{1}$, and $D_{1}$ are the classical slab quadrupole terms, but expressed in the Fourier-Laplace space:

$$
\begin{aligned}
& A_{1}=D_{1}=\cosh \left(\gamma e_{1}\right) \quad B_{1}=\frac{1}{\gamma k_{1}} \sinh \left(\gamma e_{1}\right) \quad C_{1}=\gamma k_{1} \sinh \left(\gamma e_{1}\right) \\
& X_{1}=\frac{q}{k_{1} s \gamma^{2}}\left[\cosh \left(\gamma e_{1}\right)-1\right] \quad \text { and } \quad Y_{1}=\frac{q}{s \gamma} \sinh \left(\gamma e_{1}\right)
\end{aligned}
$$


The same approach is applied to medium 2, assuming that temperature and heat flux are conservative at the interface $z=e_{1}$ :

$$
\left[\begin{array}{c}
\tau\left(\beta_{n}, e_{1}, s\right) \\
\psi\left(\beta_{n}, e_{1}, s\right)
\end{array}\right]=\left[\begin{array}{ll}
A_{2} & B_{2} \\
C_{2} & D_{2}
\end{array}\right]\left[\begin{array}{c}
\tau\left(\beta_{n}, e_{2}, s\right) \\
\psi\left(\beta_{n}, e_{2}, s\right)=0
\end{array}\right]-\left[\begin{array}{c}
X_{2} \\
Y_{2}
\end{array}\right]
$$

where index 2 indicates that the corresponding terms are calculated for medium 2 . The temperature at the interface is calculated from Eqs. (17) as

$$
\tau\left(\beta_{n}, e_{1}, s\right)=\frac{\sinh \left[\gamma\left(e_{1}+e_{2}\right)\right]}{k_{1} \cosh \left(\gamma e_{2}\right) \sinh \left(\gamma e_{1}\right)+k_{2} \cosh \left(\gamma e_{1}\right) \sinh \left(\gamma e_{2}\right)} \frac{q}{\gamma^{2} s}
$$

This expression has to be inverted. For the steady-state case, $\gamma \equiv \beta_{n}$, only inverse Fourier transform has to be performed:

$$
T\left(x, z=e_{1}\right)=\frac{2}{L} \sum_{n=0}^{\infty} \cos \left(\beta_{n} x\right) \tau\left(e_{1}, \beta_{n}\right)
$$

Equations (17) can also be used in order to determine the temperature at $z=0$ and $z=e_{2}$. As a validation test for the quadrupole extension method, this analytical solution is compared to the closest nodes corresponding to the temperature vector given by Eq. (15b). Steady-state temperature versus $x$ at $z=0$ (boundary of medium 1) and $z=e_{1}$ (interface) are ploted in Figure $3 a$. The resulting curves exhibit quite good agreement between the analytical solution and our quadrupole extension approach. The interface temperature difference between the analytical and our semianalytical solution is shown as an error plot in Figure $3 b$. This error is less than $0.25 \%$.

For clarity reasons, validation results are given only for the steady-state case, but they work as well for the transient case. A numerical Laplace inversion has to be performed, using for instance a Gavert-Stehfest algorithm [15].

\section{APPLICATION EXAMPLES: SOME PRACTICAL ASPECTS}

The numerical implementation of previous methods is very convenient with a matrix solver such as Matlab [16]. Nevertheless, several numerical problems could arise and must be tackled, and will be explained in the case of some simple application examples such as a two-temperature model, a single squared heterogeneity, and a periodic medium.

\subsection{Two-Nodes Problem or Two-Temperature Model in a Two-Phase Medium}

The two-nodes problem is both a simple and an interesting case, because the matrices and functions of matrices are relatively easy to implement with only two eigenvalues. The corresponding matrices can be studied analytically, and thus make possible a better understanding of the respective contribution of the zero eigenvalue (related to the variation of the $z$-averaged quantities into the $x$ direction) and the nonzero eigenvalue (related to the $z$-direction transfer). 


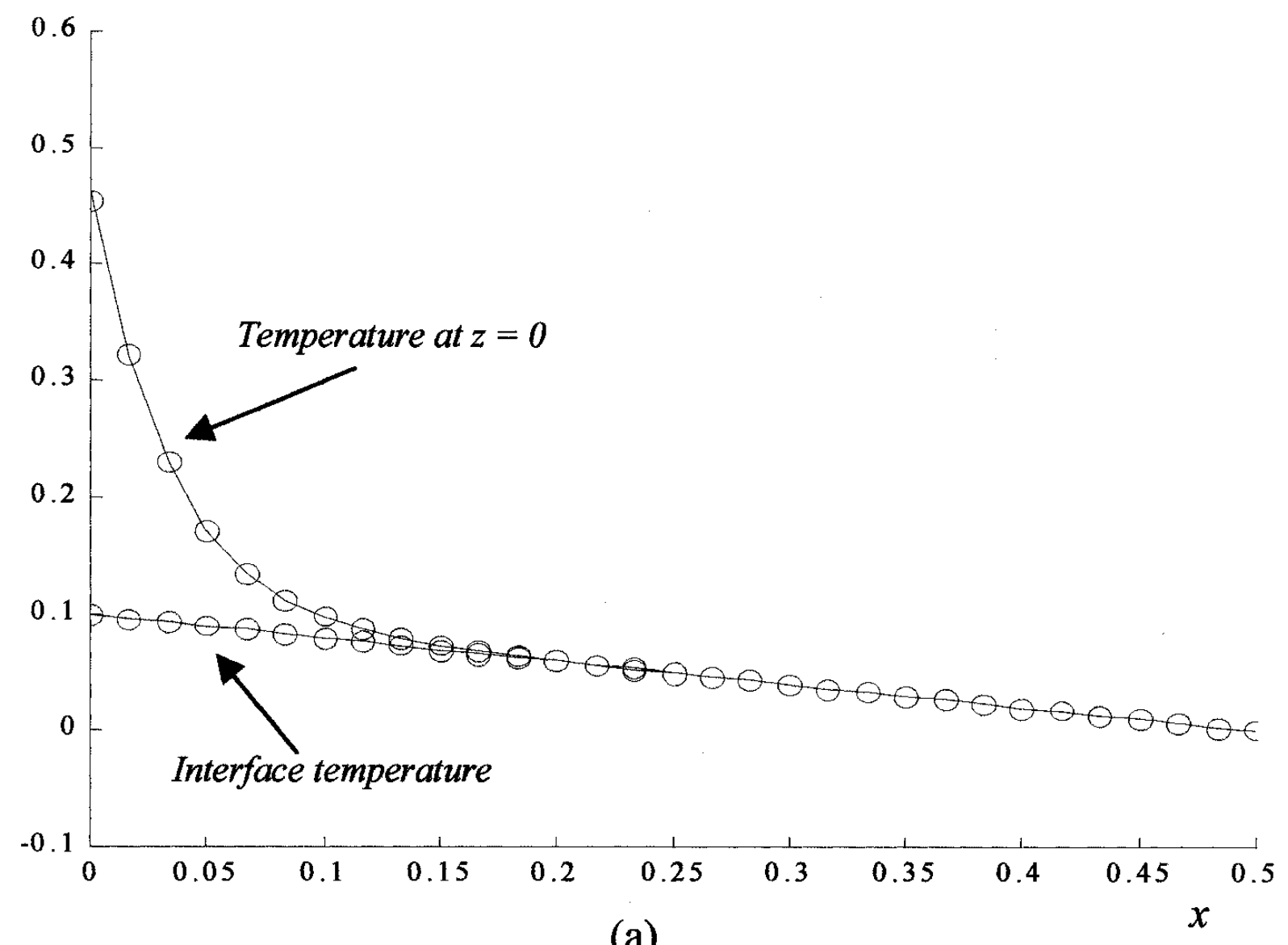

(a)

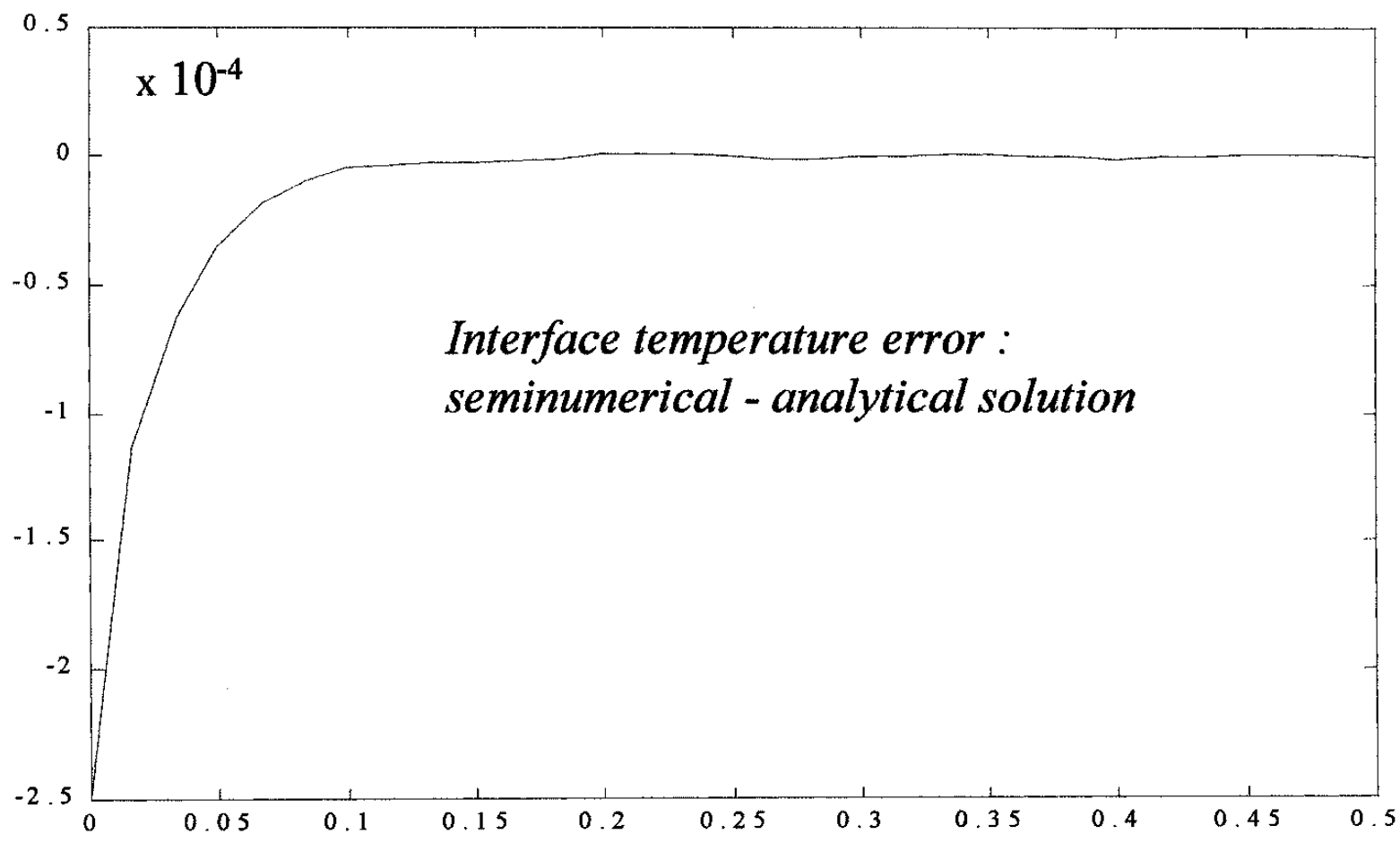

(b)

Figure 3. Temperature versus $x$, at $z=0$ and $z=e_{1}$ : (a) Comparison of analytical solution $(O)$ and quadrupole extension method, $k_{1}=0.1 \mathrm{~W} / \mathrm{m} \mathrm{K}, k_{2}=10 \mathrm{~W} / \mathrm{m} \mathrm{K}, L=0.5 \mathrm{~m}, e=0.1 \mathrm{~m}$; (b) interface temperature error plot. 
When studying heat conduction in a two-phase system, a two-equation model may be needed at the macroscopic scale when local thermal equilibrium is not fulfilled. This approach is founded on homogenization or volume-averaging techniques [17], and is helpful when the thermal properties of the two constituents differ widely, or when fast transient states are observed.

The respective macroscopic temperatures of phase 1 and phase 2, namely, $\left\langle T_{1}\right\rangle$ and $\left\langle T_{2}\right\rangle$, are solutions of the corresponding macroscopic two-equations model:

$$
\begin{aligned}
& \varepsilon_{1}(\rho c)_{1} \frac{\partial\left\langle T_{1}\right\rangle}{\partial t}=K_{11} \frac{\partial^{2}\left\langle T_{1}\right\rangle}{\partial x^{2}}-h\left(\left\langle T_{1}\right\rangle-\left\langle T_{2}\right\rangle\right) \\
& \varepsilon_{2}(\rho c)_{2} \frac{\partial\left\langle T_{2}\right\rangle}{\partial t}=K_{22} \frac{\partial^{2}\left\langle T_{2}\right\rangle}{\partial x^{2}}-h\left(\left\langle T_{2}\right\rangle-\left\langle T_{1}\right\rangle\right)
\end{aligned}
$$

with

$$
\left\langle T_{1}\right\rangle=\frac{1}{e_{1}} \int_{0}^{e_{1}} T_{1}(z) d z \quad \text { and } \quad\left\langle T_{2}\right\rangle=\frac{1}{e_{2}} \int_{0}^{e_{2}} T_{2}(z) d z
$$

Applying a Laplace transform to Eqs. (20), and writing them in a vectorial way similar to Eq. (4), makes it possible to define an equivalent matricial quadrupole form such as

$$
\mathbf{K}^{-1} \mathbf{M}_{\mathbf{e}}\left[\begin{array}{c}
\left\langle\bar{T}_{1}\right\rangle \\
\left\langle\bar{T}_{2}\right\rangle
\end{array}\right]-\frac{d^{2}\left(\left[\begin{array}{l}
\left\langle\bar{T}_{1}\right\rangle \\
\left\langle\bar{T}_{2}\right\rangle
\end{array}\right]\right)}{d x^{2}}=\mathbf{0}
$$

where

$$
\mathbf{M}_{\mathbf{e}}=\left[\begin{array}{cc}
\varepsilon_{1}(\rho c)_{1} s+h & -h \\
-h & \varepsilon_{2}(\rho c)_{2} s+h
\end{array}\right] \quad \text { and } \quad \mathbf{K}=\left[\begin{array}{cc}
K_{11} & 0 \\
0 & K_{22}
\end{array}\right]
$$

This equation could also be obtained analytically as a two-node problem from Eqs. (17a)-(17b), but the implementation of such a solution is not within the scope of the present article.

Equation (21) is similar to Eq. (4) if we replace the term $\left(\mathbf{M}_{/ /}+\mathbf{G} s\right)$ by $\mathbf{M}_{\mathbf{e}}$, with $\mathbf{F}=\mathbf{0}$. Equation (21) can be diagonalized, and the general quadrupole extension will apply. For the steady-state two-layer body as defined in the previous example (Figure 2), we have [17]

$$
\varepsilon_{1}=\frac{e_{1}}{e} \quad \varepsilon_{2}=\frac{e_{2}}{e} \quad K_{11}=\varepsilon_{1} \lambda_{1} \quad K_{22}=\varepsilon_{2} \lambda_{2} \quad h=\frac{3 \lambda_{1} \lambda_{2}}{\left(e_{2} \lambda_{1}+e_{1} \lambda_{2}\right)_{e}}
$$

and Eq. (21) is turned into

$$
\left[\begin{array}{cc}
h_{1} & -h_{1} \\
-h_{2} & h_{2}
\end{array}\right]\left[\begin{array}{c}
\left\langle T_{1}\right\rangle \\
\left\langle T_{2}\right\rangle
\end{array}\right]-\frac{d^{2}}{d x^{2}}\left(\left[\begin{array}{c}
\left\langle T_{1}\right\rangle \\
\left\langle T_{2}\right\rangle
\end{array}\right]\right)=0
$$

with $h_{i}=h / \varepsilon_{i} k_{i} ; i=1,2$. 
For that particular case, analytical diagonalization is possible, and the eigenvalues are 0 and $h_{1}+h_{2}$. According to Eq. (9) and Table 2, an intrinsic quadrupole relationship can be deduced as

$$
\left[\begin{array}{c}
\left\langle T_{1}\right\rangle_{x} \\
\left\langle T_{2}\right\rangle_{x}
\end{array}\right]=\mathbf{P}_{\mathbf{m}}\left[\begin{array}{cc}
L-x & 0 \\
0 & \frac{\sinh \left[d_{m}(L-x)\right]}{d_{m} \cosh \left(d_{m} L\right)}
\end{array}\right] \mathbf{P}_{\mathbf{m}}^{-1}\left[\begin{array}{cc}
\frac{1}{k_{1}} & 0 \\
0 & \frac{1}{k_{2}}
\end{array}\right]\left[\begin{array}{c}
q \\
q
\end{array}\right]
$$

where $\mathbf{P}_{\mathbf{m}}$ is the eigenvector matrix and $d_{m}=\sqrt{h_{1}+h_{2}}$ is the nonzero eigenvalue square root. Since this matrix is easy to calculate, a complete analytical solution can be proposed. The corresponding solution associated to the validation test case is then

$$
\left[\begin{array}{c}
\left\langle T_{1}\right\rangle_{x} \\
\left\langle T_{2}\right\rangle_{x}
\end{array}\right]=\frac{1}{k^{*} e}\left[\begin{array}{cc}
L+\frac{k_{2} e_{2} \sinh \left[d_{m}(L-x)\right]}{k_{1} e_{1} d_{m} \cosh \left(d_{m} L\right)} & L-\frac{\sinh \left[d_{m}(L-x)\right]}{d_{m} \cosh \left(d_{m} L\right)} \\
L-\frac{\sinh \left[d_{m}(L-x)\right]}{d_{m} \cosh \left(d_{m} L\right)} & L+\frac{k_{1} e_{1} \sinh \left[d_{m}(L-x)\right]}{k_{2} e_{2} d_{m} \cosh \left(d_{m} L\right)}
\end{array}\right]\left[\begin{array}{l}
q \cdot e_{1} \\
q . e_{2}
\end{array}\right]
$$

where $k^{*}$ is the one-dimensional equivalent parallel thermal conductivity such as $k^{*}=\left(k_{1} e_{1}+k_{2} e_{2}\right) / e$.

A generalized semianalytical solution such as Eq. (15a) can be integrated in order to compute the mean temperature values for both phases, and this solution is compared to the present analytical solution given by Eq. (24). The resulting curves are shown in Figure 4. Very good agreement is observed between the analytical macroscopic two-temperature model and our seminumerical quadrupole extension method.

It is important to point out that the first eigenvalue of such a system is zero, associated to the $z$-averaged quantities following the gridding direction.

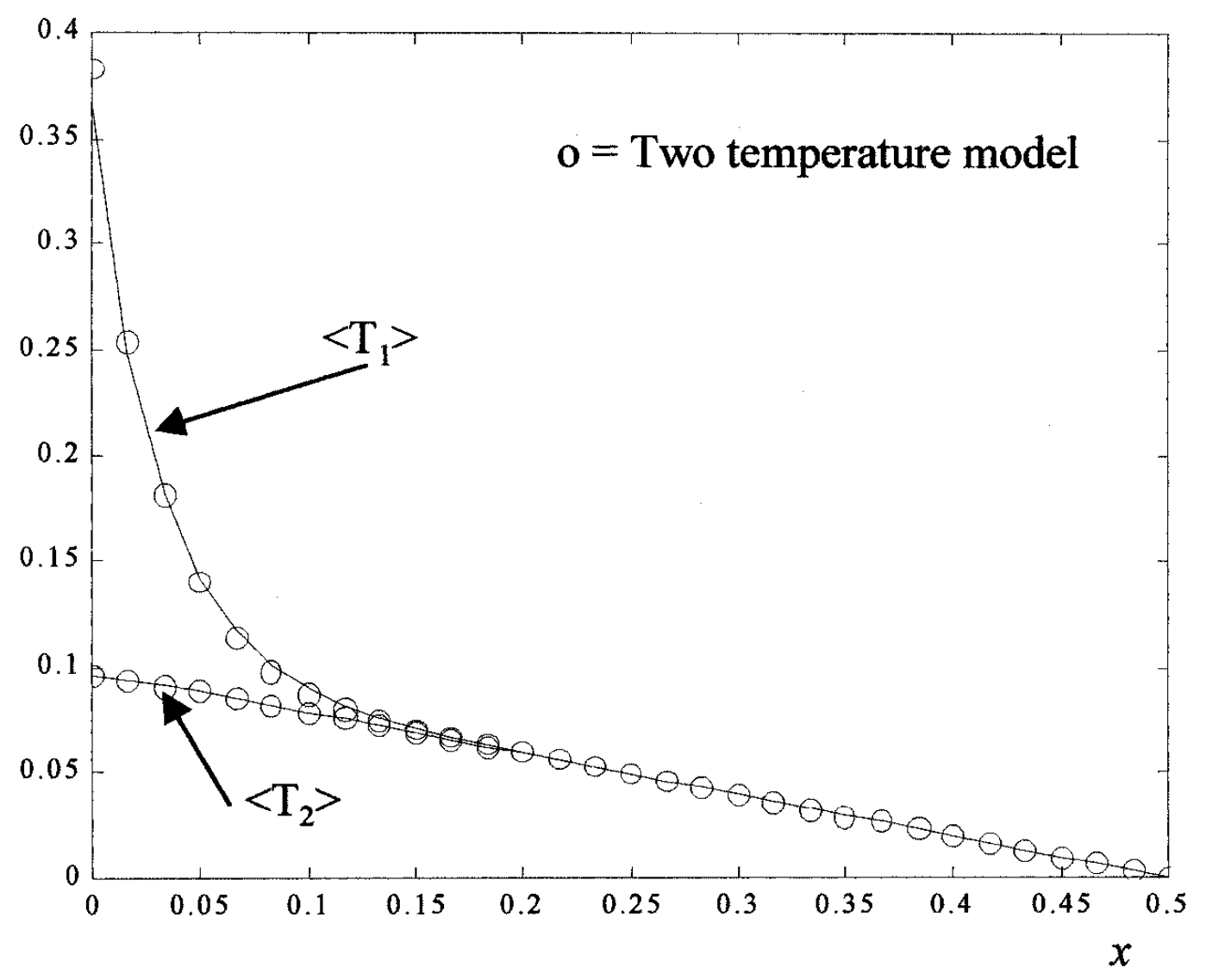

Figure 4. Steady-state temperature profile versus $x$ : Analytical two-temperature model $(\bigcirc)$ and quadrupole extension method $(M)$. 
This particular eigenvalue is relative to averaged heat transfer in the $x$ direction. On the other hand, the second eigenvalue (as well as the other eigenvalues when gridding is greater than 2 nodes) is relative to heat transfer in the $z$ direction, that is, the twodimensional effects superposed to averaged transfer. If the temperature vector is calculated at $x=L$ from Eq. (24), a hyperbolic tangent function appears. When $L$ is large, this function tends to 1 , the nonzero eigenvalue is then relative to a boundarylayer effect, independent of $L$, which can be evaluated from a thermal constriction resistance. This important point can be expanded to a more general case when gridding with a higher nodes number: if the product of a particular eigenvalue with $L$ is large enough, it is possible not to compute the corresponding hyperbolic sine or cosine functions. As shown in next section, that makes possible an eigenvalue regularization process.

\subsection{Heterogeneity Problem with Quadrupole Matrix Product and Eigenvalues Regularization}

The heterogeneous problem presented in Figure 5 can be solved by multiplying the three corresponding expanded quadrupoles obtained for the layers $L_{1}, L_{2}$, and $L_{3}$ in order to obtain a direct relationship between the temperature vector $T_{0}$ at $x=0$ and the corresponding heat flux function. In Laplace space,

$$
\left[\begin{array}{c}
\bar{T}_{0} \\
\bar{\Phi}_{0}
\end{array}\right]=\left[\begin{array}{ll}
A_{1} & B_{1} \\
C_{1} & D_{1}
\end{array}\right]\left[\begin{array}{ll}
A_{2} & B_{2} \\
C_{2} & D_{2}
\end{array}\right]\left[\begin{array}{ll}
A_{3} & B_{3} \\
C_{3} & D_{3}
\end{array}\right]\left[\begin{array}{c}
0 \\
\bar{\Phi}_{3}
\end{array}\right]
$$

where the terms of the quadrupoles are given by Eq. (9) and Table 2 as

$$
\begin{aligned}
\mathbf{A}_{\mathbf{i}} & =\mathbf{P}_{\mathbf{i}} \cosh \left(\sqrt{\mathbf{\Omega}_{\mathbf{i}}} L_{i}\right) \mathbf{P}_{\mathbf{i}}^{-1} \\
\mathbf{B}_{\mathbf{i}} & =\mathbf{P}_{\mathbf{i}} \frac{\sinh \left(\sqrt{\mathbf{\Omega}_{\mathbf{i}}} L_{i}\right)}{\sqrt{\boldsymbol{\Omega}_{\mathbf{i}}}}\left(\mathbf{K}_{\mathbf{i}} \mathbf{P}_{\mathbf{i}}\right)^{-1}
\end{aligned}
$$

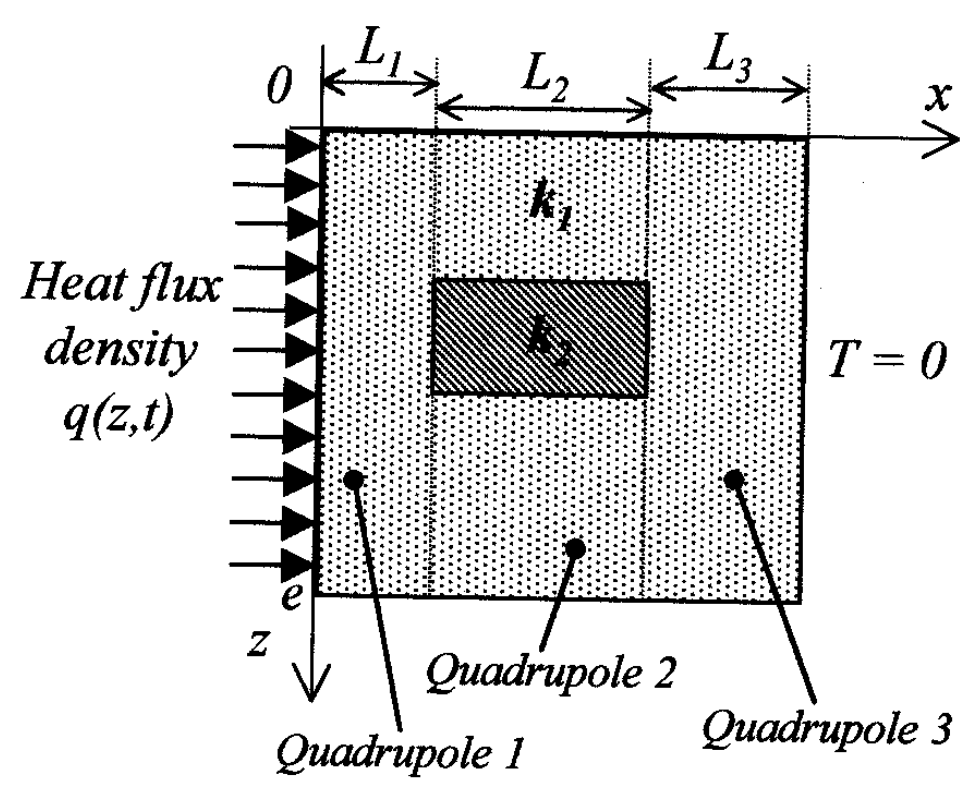

Figure 5. Single squared heterogeneity problem. 


$$
\begin{aligned}
& \mathbf{C}_{\mathbf{i}}=\mathbf{K}_{\mathbf{i}} \mathbf{P}_{\mathbf{i}} \sqrt{\boldsymbol{\Omega}_{\mathbf{i}}} \sinh \left(\sqrt{\boldsymbol{\Omega}_{\mathbf{i}}} L_{i}\right) \mathbf{P}_{\mathbf{i}}^{-\mathbf{1}} \\
& \mathbf{D}_{\mathbf{i}}=\mathbf{K}_{\mathbf{i}} \mathbf{P}_{\mathbf{i}} \cosh \left(\sqrt{\boldsymbol{\Omega}_{\mathbf{i}}} L_{i}\right)\left(\mathbf{K}_{\mathbf{i}} \mathbf{P}_{\mathbf{i}}\right)^{-1}
\end{aligned}
$$

with $i=1,2,3$. The matrices $\sqrt{\mathbf{\Omega}_{\mathbf{i}}}$ and $\mathbf{P}_{\mathbf{i}}$ are obtained from the diagonalization of the corresponding matrices $\mathbf{K}_{\mathbf{i}}\left(\mathbf{M}_{/ /, \mathbf{i}}+\mathbf{G}_{\mathbf{i}} s\right)$ defined from Eq. (4). Eliminating the flux $\boldsymbol{\Phi}_{3}$ in Eq. (25a) yields

$$
\overline{\mathbf{T}}_{\mathbf{0}}=\mathbf{M}_{\mathbf{1}} \mathbf{M}_{\mathbf{2}}^{-1} \bar{\Phi}_{\mathbf{0}}
$$

where

$$
\begin{aligned}
& \mathbf{M}_{\mathbf{1}}=\left[\mathbf{A}_{\mathbf{1}}\left(\mathbf{A}_{\mathbf{2}} \mathbf{B}_{\mathbf{3}}+\mathbf{B}_{\mathbf{2}} \mathbf{D}_{\mathbf{3}}\right)+\mathbf{B}_{\mathbf{1}}\left(\mathbf{C}_{\mathbf{2}} \mathbf{B}_{\mathbf{3}}+\mathbf{D}_{\mathbf{2}} \mathbf{D}_{\mathbf{3}}\right)\right] \\
& \mathbf{M}_{\mathbf{2}}=\left[\mathbf{C}_{\mathbf{1}}\left(\mathbf{A}_{\mathbf{2}} \mathbf{B}_{\mathbf{3}}+\mathbf{B}_{\mathbf{2}} \mathbf{D}_{\mathbf{3}}\right)+\mathbf{D}_{\mathbf{1}}\left(\mathbf{C}_{\mathbf{2}} \mathbf{B}_{\mathbf{3}}+\mathbf{D}_{\mathbf{2}} \mathbf{D}_{\mathbf{3}}\right)\right]
\end{aligned}
$$

The main problem arising is the possible ill conditioning number of some of the quadrupole terms given by Eq. (25c), especially when the layer length $L_{i}$ is large or when the dimension of the matrices is large (high number of discretized temperatures). It is then necessary to regularize the eigenvalues. The eigenvalues corresponding to the three layers $(i=1,2,3)$ are plotted in Figure 6 , in the steady-state case, and with adiabatic boundary conditions at $z=0$ and $z=e$.

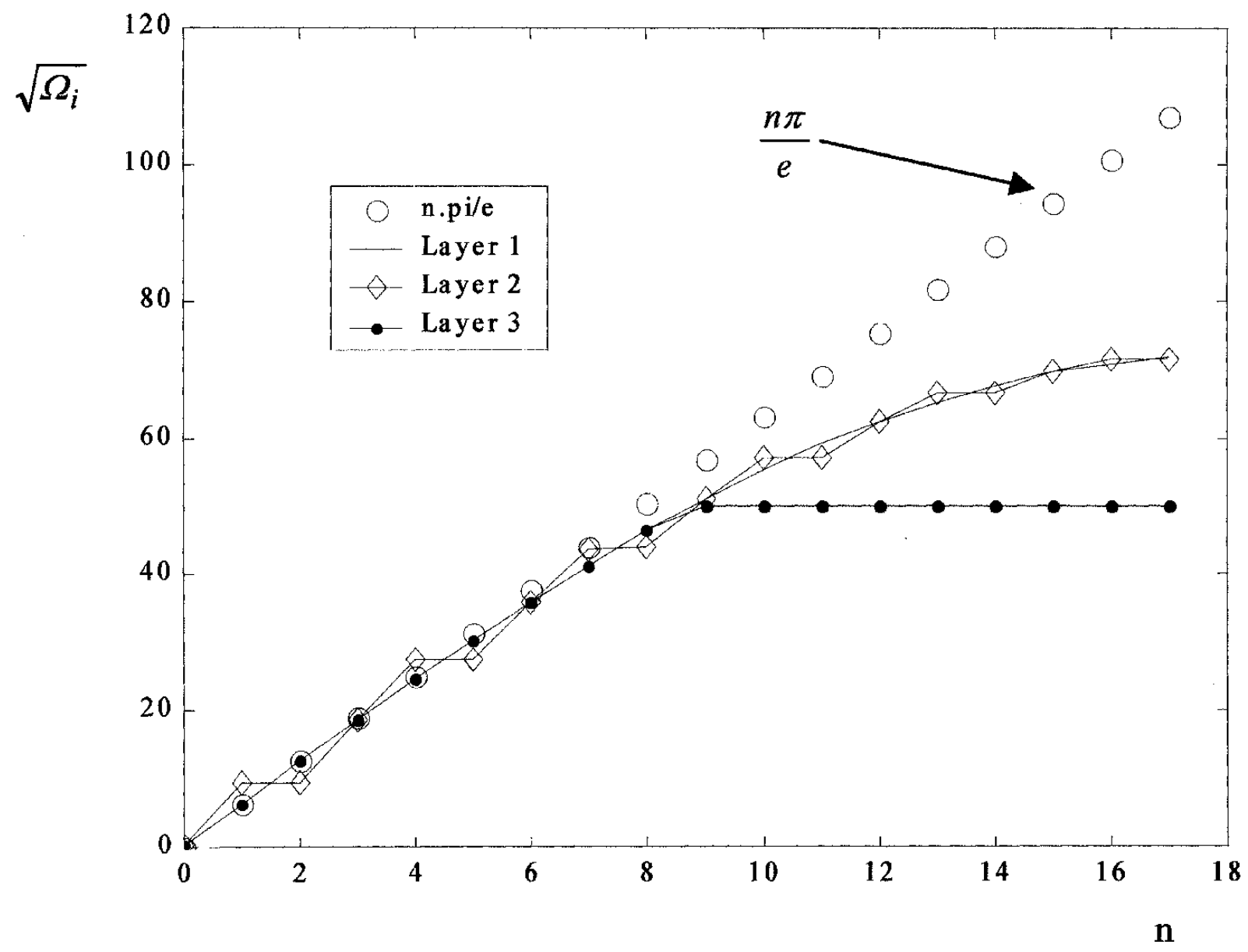

Figure 6. Eigenvalues regularization: $L_{1}=0.01, L_{2}=0.1, L_{3}=0.2$. 
It is important to point out that the square roots of the eigenvalues are quite near to the previous $\beta_{n}=n \pi / e$ terms, that is, to the corresponding homogeneous medium boundary-value problem. This is true only in the steady-state case. For transient state, the diagonal terms introduced by the Laplace variable $s$ would imply a different behavior. Due to spacial discretization, the eigenvalues exhibit an aliasing effect. For layer 2, small variations due to the local variation of thermal conductivity are observed. Layer 3 has to be regularized, due to its greater length. We used a truncation of the spectrum of the eigenvalues, which is close to a singular value decomposition. This regularization of the highest eigenvalues does not introduce an important bias, because a thick medium is a natural filter for high frequencies, $\sqrt{\Omega_{i}} L_{i} \leq 10$.

Steady-state temperature profiles are shown in Figure 7 for various geometric conditions. When the thermal conductivity contrast is lower, or the first layer thickness is higher, this temperature profile is smooth, and it is difficult to "see" the heterogeneity. However, the mean temperature $\left\langle\mathbf{T}_{\mathbf{0}}\right\rangle$ would contain the information relative to this heterogenity, and a global thermal resistance could be used. The dash lined curve shows how a thin heterogeneity is "seen" through a thick first layer. The curves plotted with stars or diamonds are obtained thanks to an eigenvalue regularization in the second layer, due to their higher length $L_{2}$ value.

The corresponding model remains valid for any thermal properties profile, in any of the three layers, either smooth or straight. Of particular interest is the study of such smooth cases for the implementation of inverse methods for thermal nondestructive evaluation techniques.

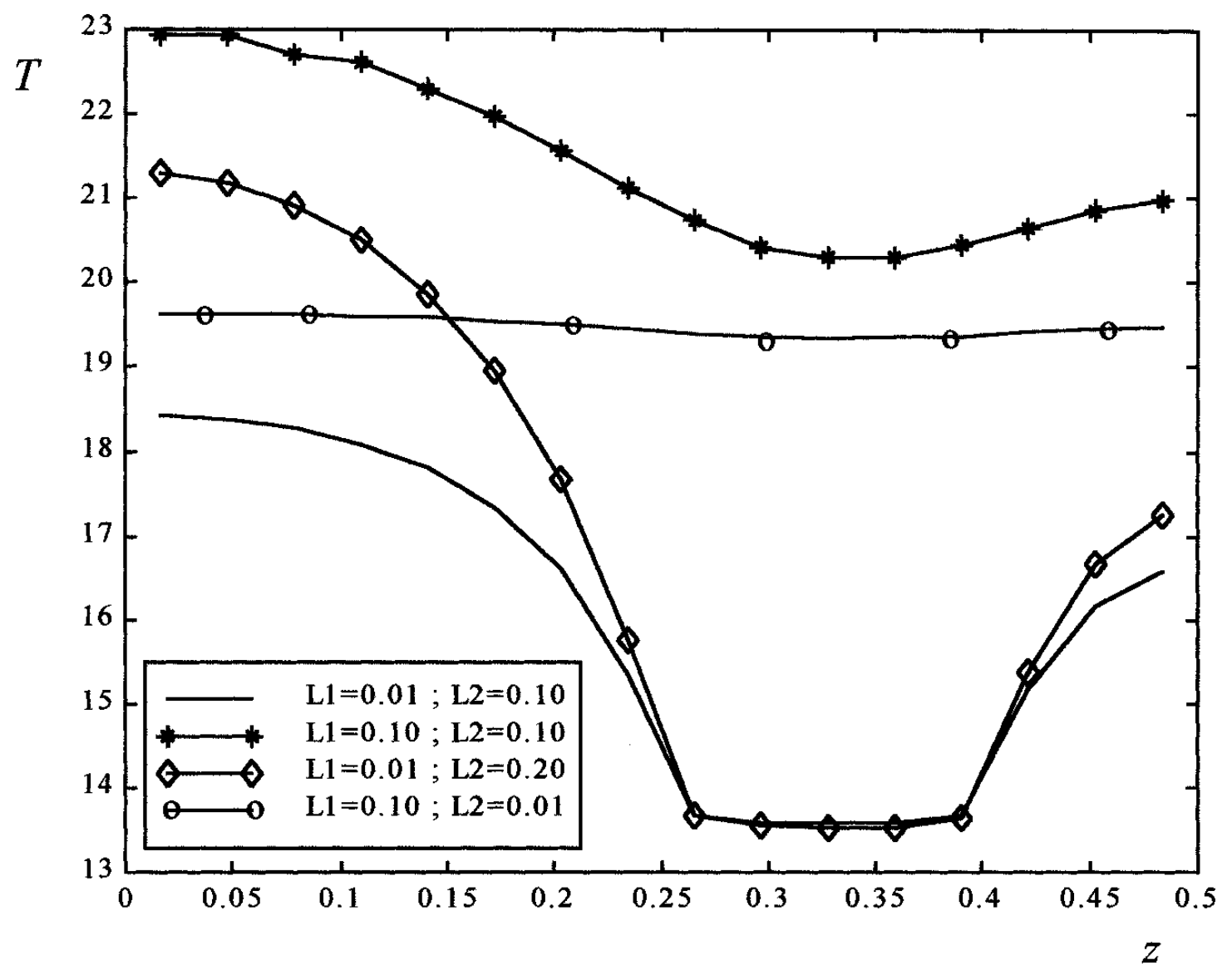

Figure 7. Temperature at $x=0$ versus $z: L_{3}=0.2, k_{1}=0.1, k_{2}=10, N=18, e=0.5 \mathrm{~m}$. 


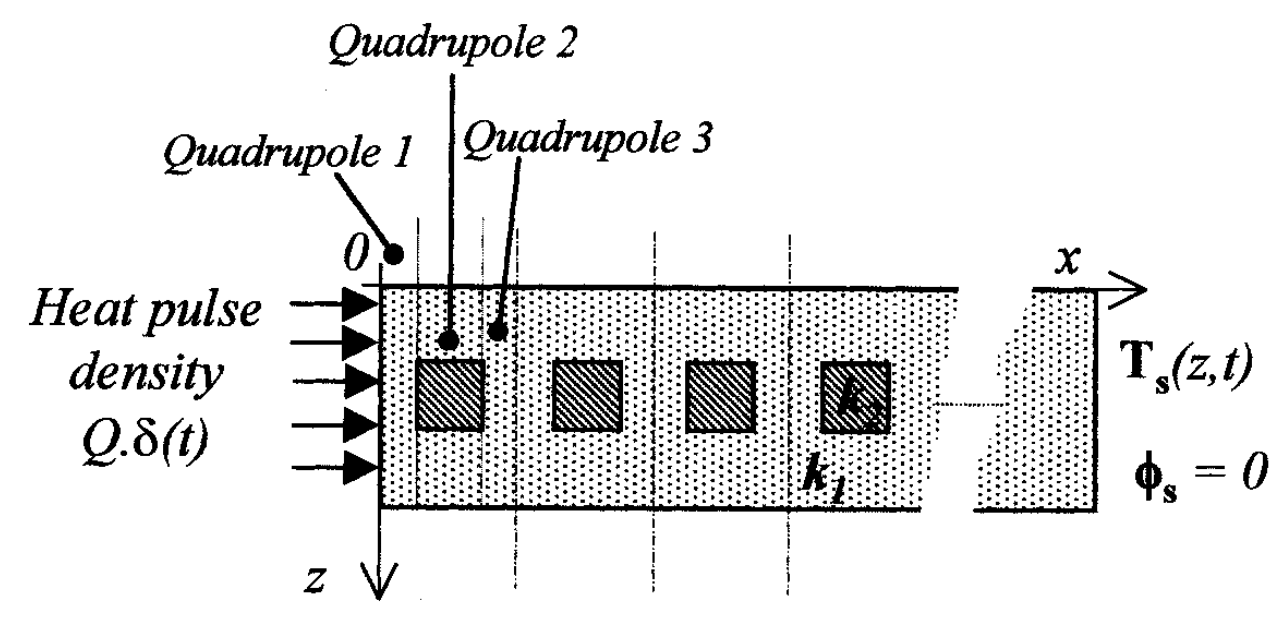

Figure 8. Periodic medium.

\subsection{Periodic Medium in Transient State}

The last example shows how simple the implementation of the method is in the case of a periodic two-dimensional porous medium. Equation $(25 a)$ can be generalized by the simple use of power of matrices obtained in the previous example. For the transient case presented in Figure 8, this yields

$$
\left[\begin{array}{l}
\overline{\mathbf{T}}_{\mathbf{0}} \\
\overline{\boldsymbol{\Phi}}_{\mathbf{0}}
\end{array}\right]=\left(\left[\begin{array}{ll}
A_{1} & \mathbf{B}_{1} \\
\mathbf{C}_{1} & \mathbf{D}_{1}
\end{array}\right]\left[\begin{array}{ll}
A_{2} & \mathbf{B}_{2} \\
\mathbf{C}_{2} & \mathbf{D}_{2}
\end{array}\right]\left[\begin{array}{ll}
A_{3} & B_{3} \\
C_{3} & D_{3}
\end{array}\right]\right)^{m}\left[\begin{array}{c}
\overline{\mathbf{T}}_{\mathbf{s}} \\
\mathbf{0}
\end{array}\right]
$$

where $m$ is the blocks number and $\bar{\Phi}_{0}=Q \cdot\left[\begin{array}{lll}\Delta z_{1} & \cdots & \Delta z_{N}\end{array}\right]^{t}$.

Such an expression is time-consuming when the number $m$ of cells is great, but the implementation with a matrix solver such as Matlab is very direct and convenient. The numerical Laplace inversion is performed using a Gavert-Stehfest algorithm [15]. The results, shown in Figure 9, are computed from Eqs. (25b) and (26). For thin layers, Eq. (12) can also apply. A significant CPU time gain results, as diagonalization is no longer necessary.

Figure $9 a$ shows temperature profile versus the $z$ direction for different values of layer number $m$ at time $t=2 \mathrm{~s}$. This time was chosen as near to the half rising point of the temperature. The total length is the same for all values of $m$. Rear face averaged temperatures are ploted in Figure $9 b$ as a function of time, for various values of $m$. These curves show how the medium tends to be similar to an equivalent homogenized medium as $m$ is incremented.

\section{CONCLUSION}

An extension of the thermal quadrupole formalism has been proposed for heat conduction modeling in heterogeneous media, when the variation of thermal properties is one-dimensional. The main problem was to find a generalized intrinsic relationship between temperature and heat flux at the boundaries of an heterogeneous medium. A semianalytical general solution was found for two-dimensional transient heat transfer in finite or semi-infinite media in both axial and radial coordinate systems. The extension of the basic thermal quadrupole formalism was obtained from the one-dimensional discretization in the properties-variation 


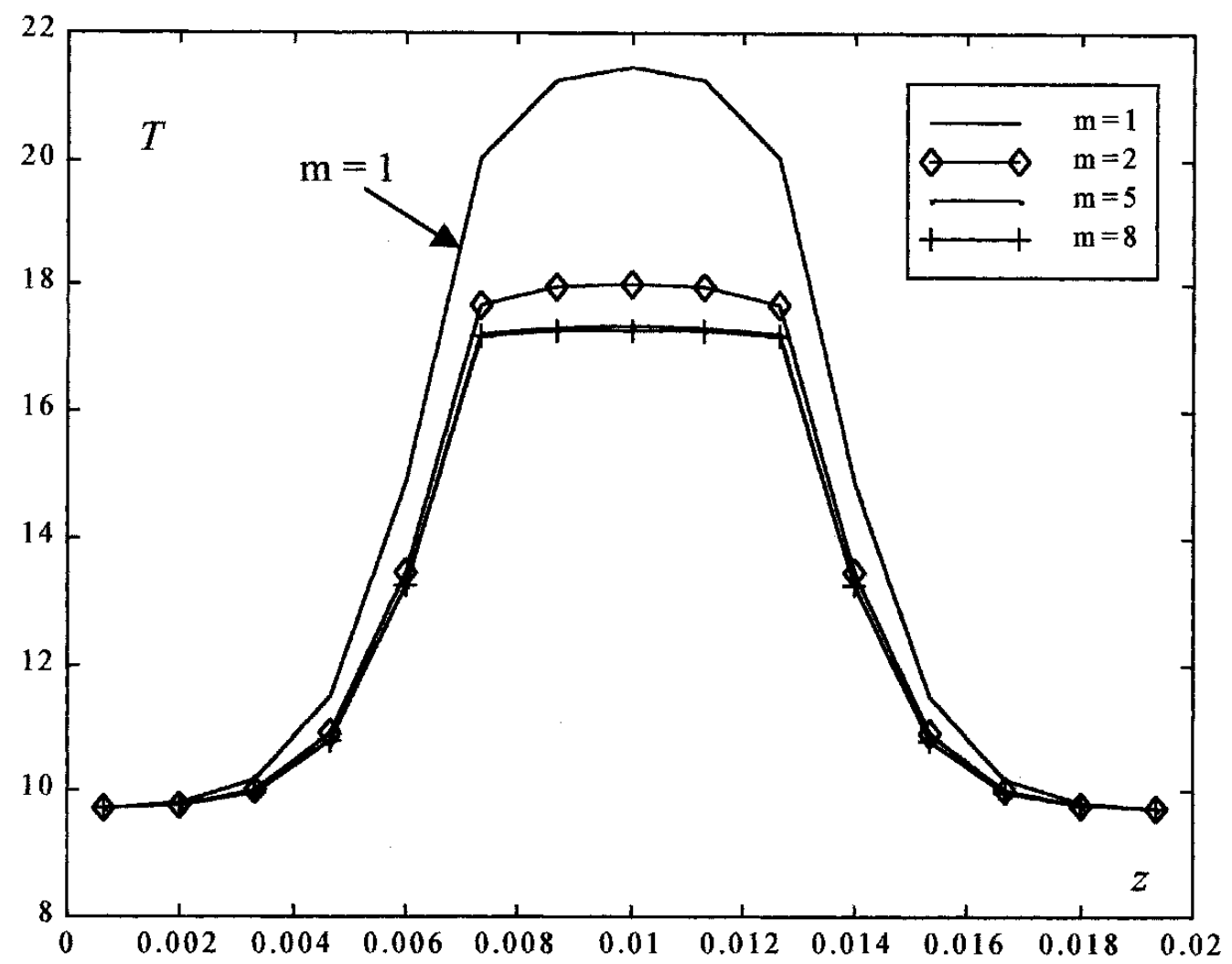

(a)

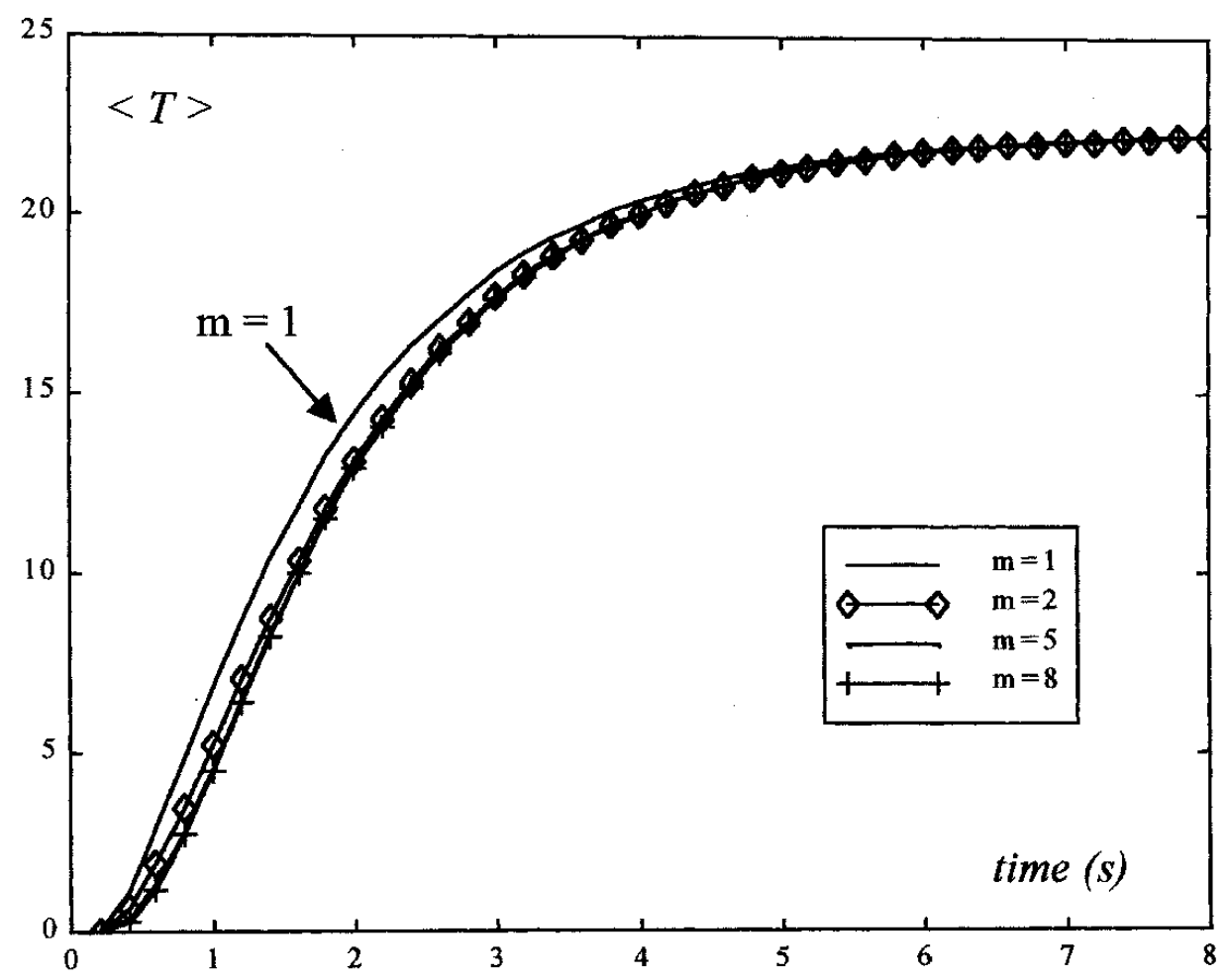

(b)

Figure 9. Rear face response to front heat pulse for different values of $m$ : $(a)$ Temperature versus $z$ for $a_{1} t / L^{2}=0.125 ;(b) z$-averaged temperature versus time, $L_{1}=0.001 / \mathrm{m}, L_{2}=0.002 / \mathrm{m}, L_{3}=0.001 / \mathrm{m}$, $k_{1}=0.1, k_{2}=10$. 
direction, coupled with semianalytical solution of the corresponding vectorial differential equation. The generalization of the proposed method to a three-dimensional case is relatively easy, and two-dimensional gridding is then applied.

This approach is of great interest when dealing with semi-infinite or multilayered media. One advantage is that complex heterogeneities can be modeled with simple matrix products, as allowed for homogeneous media with the classical thermal quadrupole formalism. Another gain is that not gridding in the infinite or semiinfinite case could bring a significant simplification. The transient case treatment, based on Laplace transform, cancels any accumulation of errors associated with a time-step approach. Furthermore, the semianalytical formalism is adapted to asymptotic expansion analysis for example in the case of infinitely thin media. Thus, this kind of approach is suitable for solving inverse problems such as those found when dealing with thermal property measurement in heterogeneous media [18].

Some practical aspects of the use of the resulting functions of matrices have been explained in the case of simple application examples such as a two-layered medium and a single squared heterogeneity in two-dimensional steady state, and a periodic medium in transient state. The ill conditioning in several cases has been avoided by a regularization method.

\section{REFERENCES}

1. A. Degiovanni, Conduction dans un "mur" multicouche avec sources: Extension de la notion de quadripôle, Int. J. Heat. Mass. Transfer, vol. 3, pp. 553-557, 1988.

2. J. C. Batsale, D. Maillet, and A. Degiovanni, Extension de la notion de quadripole thermique à l'aide de transformations intégrales: Calcul du transfert thermique au travers d'un défaut plan bidimensionnel, Int. J. Heat. Mass. Transfer, vol. 37, pp. 111-127, 1994.

3. C. Aviles-Ramos, A. Haji-Sheikh, and J. V. Beck, Exact Solution of Heat Conduction in Composite Materials and Application to Inverse Problems, ASME J. Heat Transfer, vol. 120, pp. 592-599, 1998.

4. L. Yan, A. Haji-Sheikh, and J. V. Beck, Thermal Characteristics of Two-Layered Bodies with Embedded Thin-Film Heat Source, ASME J. Electron. Pkging. vol. 115, no. 3, pp. 276-283, 1993.

5. C. W. Tittle, Boundary Value Problems in Composite Media, J. Appl. Phys., vol. 36, no. 4, pp. $1486-1488,1965$.

6. N. Ozisik, Heat Conduction, 2nd ed., Wiley, New York, 1993.

7. Ph. Leturk, J. M. Dorkel, F. E. Ratolojanahary, and S. Tounsi, A Two-Port Network Formalism for 3D Heat Conduction in Multilayered Media, Int. J. Heat Mass Transfer, vol. 36, no. 9, pp. 2317-2326, 1993.

8. C. A. Brebbia, J. C. F. Telles, and L. C. Wrobel, Boundary Element Techniques: Theory and Application in Engineering, Springer-Verlag, Berlin, 1984.

9. R. Pasquetti and A. Caruso, Boundary Element Approach for Transient and Nonlinear Thermal Diffusion, Numer. Heat Transfer B, vol. 17, pp. 83-99, 1990.

10. D. Mourand, J. Gounot, and J. C. Batsale, New Sequential Method to Process Noisy Temperature Response from Flash Experiment Measured by Infrared Camera, Rev. Sci. Instrum., vol. 69, no. 3, pp. 1437-1440, 1998.

11. S. V. Patankar, Numerical Heat Transfer and Fluid Flow, Hemisphere, New York, 1980.

12. X. Zhang, Métrologie par méthode quasi-instationnaire: Modélisation, identification, et application à la caractérisation de solides, Thèse de doctorat, INPL, 1993. 
13. D. Maillet, S. André, J. C. Batsale, A. Degiovanni, and C. Moyne, Thermal Quadrupoles: Solving the Heat Equation Through Integral Transforms, Wiley, New York, 2000.

14. C. B. Moler and C. F. Van Loan, Nineteen Dubious Ways to Compute the Exponential of a Matrix, SIAM Rev., vol. 20, pp. 801-836, 1979.

15. H. Stehfest, Remark on Algorithm 368: Numerical Inversion of Laplace Transform, A.C.M., vol. 53, no. 10, p. 624, 1970.

16. MATLAB User's Guide, MathWorks, South Natick, MA, 1998.

17. M. Quintard and S. Whitaker, One and Two-Equation Models for Transient Diffusion Processes in Two-Phase Systems, Adv. Heat Transfer, vol. 23, pp. 369-464, 1993.

18. B. Ladevie, J. C. Batsale, and O. Fudym, A New Simple Device to Estimate Thermophysical Properties of Insulating Materials, Int. Commun. Heat Mass Transfer, vol. 27, no. 4, pp. 473-484, 2000. 